\title{
The Sex-Specific VC Neurons Are Mechanically Activated Motor Neurons That Facilitate Serotonin-Induced Egg Laying in $C$. elegans
}

\author{
${ }^{\circledR}$ Richard J. Kopchock, III, ${ }^{1}{ }^{\circledR}$ Bhavya Ravi, ${ }^{1,2}$ Addys Bode, ${ }^{1}$ and ${ }^{\circledR}$ Kevin M. Collins ${ }^{1,2}$ \\ ${ }^{1}$ Department of Biology, University of Miami, Coral Gables, Florida 33143, and ${ }^{2}$ Neuroscience Program, University of Miami School of Medicine, \\ Miami, Florida 33136
}

Successful execution of behavior requires coordinated activity and communication between multiple cell types. Studies using the relatively simple neural circuits of invertebrates have helped to uncover how conserved molecular and cellular signaling events shape animal behavior. To understand the mechanisms underlying neural circuit activity and behavior, we have been studying a simple circuit that drives egg-laying behavior in the nematode worm Caenorhabditis elegans. Here we show that the sex-specific, ventral C (VC) motor neurons are important for vulval muscle contractility and egg laying in response to serotonin. $\mathrm{Ca}^{2+}$ imaging experiments show the VCs are active during times of vulval muscle contraction and vulval opening, and optogenetic stimulation of the VCs promotes vulval muscle $\mathrm{Ca}^{2+}$ activity. Blocking VC neurotransmission inhibits egg laying in response to serotonin and increases the failure rate of egg-laying attempts, indicating that VC signaling facilitates full vulval muscle contraction and opening of the vulva for efficient egg laying. We also find the VCs are mechanically activated in response to vulval opening. Optogenetic stimulation of the vulval muscles is sufficient to drive $\mathrm{VC} \mathrm{Ca}^{2+}$ activity and requires muscle contractility, showing the presynaptic VCs and the postsynaptic vulval muscles can mutually excite each other. Together, our results demonstrate that the VC neurons facilitate efficient execution of egg-laying behavior by coordinating postsynaptic muscle contractility in response to serotonin and mechanosensory feedback.

Key words: ACh; behavior; circuit; feedback; mechanosensation; serotonin

Significance Statement

Many animal motor behaviors are modulated by the neurotransmitters, serotonin and ACh. Such motor circuits also respond to mechanosensory feedback, but how neurotransmitters and mechanoreceptors work together to coordinate behavior is not well understood. We address these questions using the egg-laying circuit in Caenorhabditis elegans where we can manipulate presynaptic neuron and postsynaptic muscle activity in behaving animals while recording circuit responses through $\mathrm{Ca}^{2+}$ imaging. We find that the cholinergic VC motoneurons are important for proper vulval muscle contractility and egg laying in response to serotonin. Muscle contraction also activates the VCs, forming a positive feedback loop that promotes full contraction for egg release. In all, mechanosensory feedback provides a parallel form of modulation that shapes circuit responses to neurotransmitters.

Received Aug. 14, 2020; revised Dec. 8, 2020; accepted Mar. 1, 2021.

Author contributions: R.J.K., B.R., and K.M.C. designed research; R.J.K. and B.R. performed research; R.J.K., A.B., and K.M.C. contributed unpublished reagents/analytic tools; R.J.K., B.R., and K.M.C. analyzed data; R.J.K. wrote the first draft of the paper; R.J.K., B.R., and K.M.C. edited the paper; R.J.K. wrote the paper.

This work was supported by National Science Foundation Grants R01-NS086932 and IOS-1844657 to K.M.C. R.J.K. was supported by a University of Miami Maytag Fellowship. Some of the strains used in this study were provided by the C. Elegans Genetics Center, which is funded by National Science Foundation Office of Research Infrastructure Programs (P40 0D010440). We thank David M. Miller, III, for sharing plasmids; and Drs. Julia Dallman, Grace Zhai, and Mason Klein, and members of the K.M.C. laboratory for helpful discussions and feedback on the manuscript.

The authors declare no competing financial interests.

Correspondence should be addressed to Keven M. Collins at kevin.collins@miami.edu.

https://doi.org/10.1523/JNEUROSCI.2150-20.2021

Copyright $\odot 2021$ the authors

\section{Introduction}

A fundamental goal of neuroscience is to understand the neural basis of behavior (Bargmann and Marder, 2013). Recent work reporting the synaptic wiring diagrams, or connectomes, of nervous systems provides an unprecedented opportunity to study how the nervous system directs animal behavior (Lerner et al., 2016; Meinertzhagen, 2018; Cook et al., 2019). However, connectomes alone are not sufficient to predict nervous system function (Swanson and Lichtman, 2016; Batista-García-Ramó and Fernández-Verdecia, 2018; Taylor et al., 2019). Released neurotransmitters can signal both synaptically and extrasynaptically through distinct receptors to drive short-term and long-term behavior changes (Chase et al., 2004; Hardingham and Bading, 
2010; Donnelly et al., 2013; Del-Bel and De-Miguel, 2018; Koelle, 2018). Neuropeptides can be coreleased from synapses and signal alongside neurotransmitters (Nusbaum et al., 2017; Brewer et al., 2019). Understanding how an assembly of ionotropic and metabotropic signaling events drives the complex pattern of circuit activity is facilitated by direct tests in invertebrate animals (Bargmann and Marder, 2013), such as those amenable to genetic investigation (Sengupta and Samuel, 2009). Such studies have the potential to reveal conserved neural circuit signaling mechanisms that underlie behavior.

The egg-laying circuit of the nematode worm Caenorhabditis elegans provides an ideal model for such a reductionist approach (see Fig. $1 A, B$ ). The canonical egg-laying circuit is comprised of three main cell types: the serotonergic hermaphrodite-specific neuron (HSN) command motor neurons, the vulval muscles, and the cholinergic ventral C (VC) motor neurons (Schafer, 2006). An active egg-laying behavior state is initiated when the HSN neurons release serotonin (Desai et al., 1988; Waggoner et al., 1998), which signals through $G$ protein-coupled serotonin receptors (Hapiak et al., 2009; Fernandez et al., 2020) and NLP-3 neuropeptides (Brewer et al., 2019). Individual eggs are laid when the vm1 and vm2 vulval muscle cells contract in synchrony to open the vulva (Collins and Koelle, 2013; Li et al., 2013). The $\mathrm{VC}$ motor neurons are the primary cholinergic neurons of the egg-laying circuit (Duerr et al., 2001; Pereira et al., 2015) and are active during egg-laying behavior (Zhang et al., 2008; Collins et al., 2016), but the function of their signaling is not well understood (Schafer, 2006). As in mammals, most muscle contraction events in C. elegans are $\mu$ ltimately driven by ACh (Richmond and Jorgensen, 1999; Trojanowski et al., 2016). nAChR agonists promote egg laying by acting on the vulval muscles (Waggoner et al., 2000; Kim et al., 2001), consistent with the VCs and/or other motor neurons releasing ACh to excite the vulval muscles (Waggoner et al., 1998). However, ACh synthesis and packaging mutants are hyperactive for egg laying, indicating that ACh can also inhibit egg laying, possibly by signaling through inhibitory muscarinic ACh receptors (Bany et al., 2003; Fernandez et al., 2020). This hyperactive egg-laying phenotype resembles animals in which VC neuron development has been disrupted by laser ablation or mutation (Bany et al., 2003), suggesting instead that ACh released from the VCs acts, at least in part, to inhibit egg laying, perhaps in response to sensory input. The VCs extend processes along the vulva, leading to the proposal they might also relay mechanosensory feedback in response to vulval opening (Zhang et al., 2010). How the VC neurons become activated and signal to regulate egg-laying behavior remains unclear. Such insight is necessary for each cell in a neural circuit to transform static information from wiring diagrams into dynamic and meaningful understanding of animal behavior.

Here we address the function of the VC neurons during egglaying behavior. We find that the VCs function within a serotonergic pathway to drive egg release. The VCs provide excitatory input to convert the initial stages of vulval muscle contraction into a successful egg-laying event. The VCs achieve this through direct activation in response to vulval muscle excitation and contraction, forming a positive feedback loop until successful egg laying is achieved.

\section{Materials and Methods}

\section{Nematode culture and strains}

All C. elegans strains were maintained at $20^{\circ} \mathrm{C}$ on Nematode Growth Medium (NGM) agar plates seeded with OP50 Escherichia coli as described previously (Brenner, 1974). All assays were conducted on agematched adult hermaphrodites at $24-30 \mathrm{~h}$ past the late L4 stage, unless otherwise stated. A list of all strains generated and used in this study can be found in Table 1.

\section{Plasmid and strain construction}

Oligonucleotides were synthesized by IDT-DNA. PCR was performed using high-fidelity Phusion DNA polymerase (New England Biolabs), except for routine genotyping, which was performed using standard Taq DNA polymerase. Plasmids were prepared using a QIAGEN miniprep spin kit. DNA concentrations were determined using a Nano-Drop spectrophotometer.

\section{Tetanus toxin ( $T e T x$-expressing transgenes}

$V C$ neuron TeTx. A $\sim 1.4 \mathrm{kB}$ DNA fragment encoding TeTx was cut from pAJ49 (Jose et al., 2007) with AgeI/XhoI, and ligated into a similarly digested pKMC145 [lin-11::GFP::unc-54 3' UTR] to generate

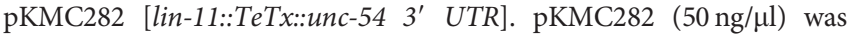
injected along with pL15EK $(50 \mathrm{ng} / \mu \mathrm{l})$ (Clark et al., 1994) into LX1832 lite-1(ce314) lin-15(n765ts) $X$ generating four independent transgenic lines of which one, MIA113 keyEx32 [lin-11::TeTx::unc-54 3'UTR + lin15(+)]; lite-1(ce314) lin-15(n765ts) X, was used for integration. keyEx32 was integrated with UV/TMP generating three independent integrants keyIs32-33 and keyIs46 [lin-11::TeTx::Unc-54 3'UTR + lin-15(+)]. Each transgenic line was backcrossed 6 times to LX1832 generating strains MIA144-146. All transgenic strains appeared phenotypically similar, and MIA144 and MIA146 were used for experiments and further crosses. To eliminate HSNs in animals lacking VC synaptic transmission, MIA26 egl-1 $(n 986 \mathrm{dm}) \mathrm{V}$ mutant animals were crossed with MIA146 to generate MIA173 keyIs46; egl-1(n986dm) V; lite-1(ce314) lin-15(n765ts) X.

Histamine-gated chloride channel ( $\mathrm{HisCl}$-expressing transgenes

$V C$ neuron HisCl. The $\sim 1.3 \mathrm{kB}$ DNA fragment encoding Drosophila HisCl HisCl1 was PCR amplified from pNP403 (Pokala et al., 2014) using the oligonucleotides $5^{\prime}$-GCG CCC GGG GTA GAA AAA ATG CAA AGC CCA ACT AGC AAA TTG G-3' and 5'-GCG GAG CTC TTA TCA TAG GAA CGT TGT CCA ATA GAC AAT A-3', cut with $\mathrm{XmaI} / \mathrm{SacI}$, and ligated into AgeI/SacI-digested pKMC145 to generate

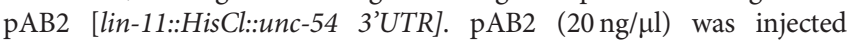
into LX1832 along with pL15EK (50 ng/ $\mu \mathrm{l})$, generating the extrachromosomal line MIA93 keyEx24 [lin-11::HisCl::unc-54 3'UTR + lin-15(+)]; lite-1(ce314) lin-15(n765ts) X. The extrachromosomal transgene subsequently integrated using UV/TMP to generate the transgenes keyIs23-30 [lin-11::HisCl::unc-54 3'UTR + lin-15(+)]. Strains bearing these transgenes were then backcrossed to the LX1832 parent strain 6 times, generating strains MIA124, MIA125, MIA130, MIA131, and MIA132. All transgenic strains appeared phenotypically similar, and MIA125 was used for experiments and further crosses. To eliminate the HSN neurons in animals expressing $\mathrm{HisCl}$ in the $\mathrm{VC}$ neurons, MIA125 keyIs23; lite-1 (ce314) lin-15(n765ts) $X$ was crossed with MIA26 to generate MIA176 keyIs23; egl-1(n986dm); lite-1(ce314) lin-15(n765ts) X.

\section{Channelrhodopsin-2 (ChR2)-expressing transgenes}

Vulval muscle ChR2. To express ChR2 in the vulval muscles, the $\sim 1$ kB DNA fragment encoding for ChR2 was PCR amplified from pRK7 [del-1::ChR2(H34R/T159C::Unc-54 3' UTR] using oligonucleotides 5' GCG GCT AGC ATG GAT TAT GGA GGC GCC CTG-3' and 5'-GCG GGT ACC TCA GGT GGC CGC GGG GAC CGC GCC AGC CTC GGC C-3'. The amplicon and recipient plasmid pBR3 (Ravi et al., 2018a) were digested with NheI/KpnI, generating pRK11 [ceh-24::ChR2

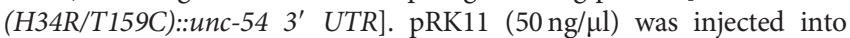
LX1832 along with pL15EK (50 ng/ $\mu \mathrm{l})$ generating MIA212 keyEx43 [ceh-24::ChR2(H34R/T159C)::unc-54 3'UTR + lin-15(+)], which was subsequently integrated with UV/TMP, generating five independent integrated transgenes keyIs47-51. Strains carrying these integrated transgenes were then backcrossed to the LX1832 parent strain 6 times, generating the strains MIA229-232 and MIA242. All transgenic strains were phenotypically similar, and MIA229 was used for experiments and further crosses. 
A
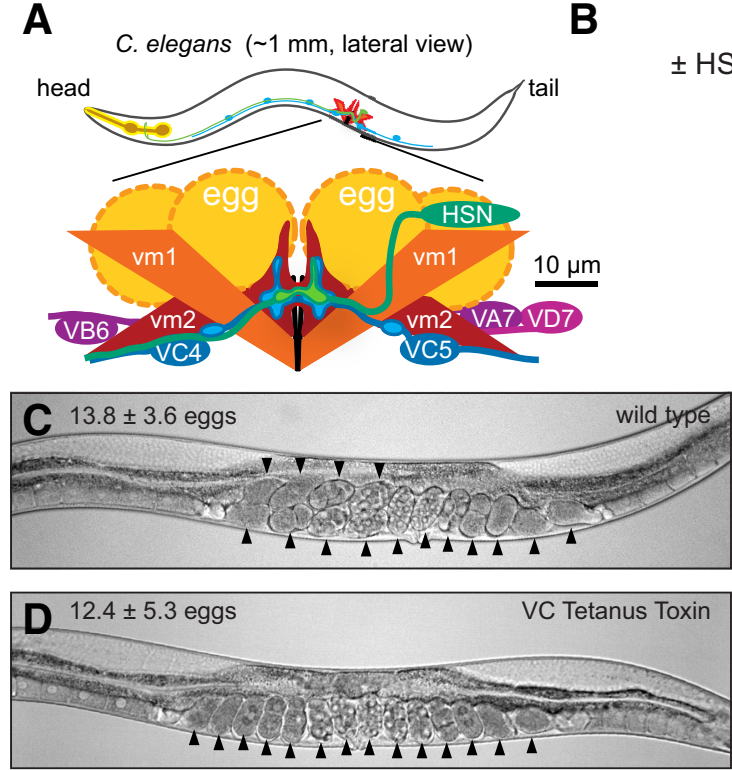

G

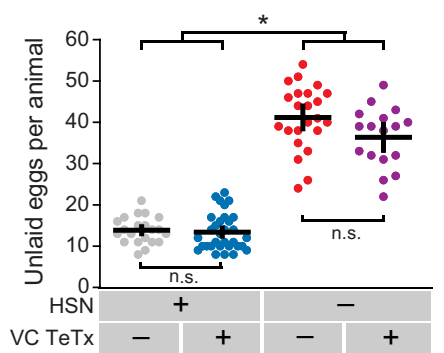

H
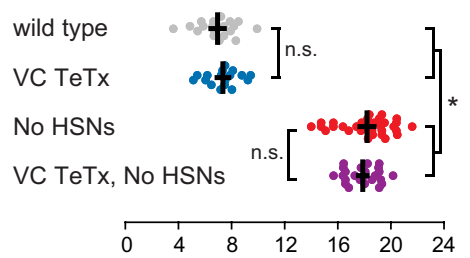
Time of first egg laid $(\mathrm{h})$

I

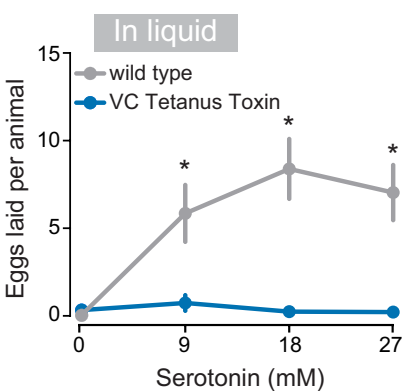

J
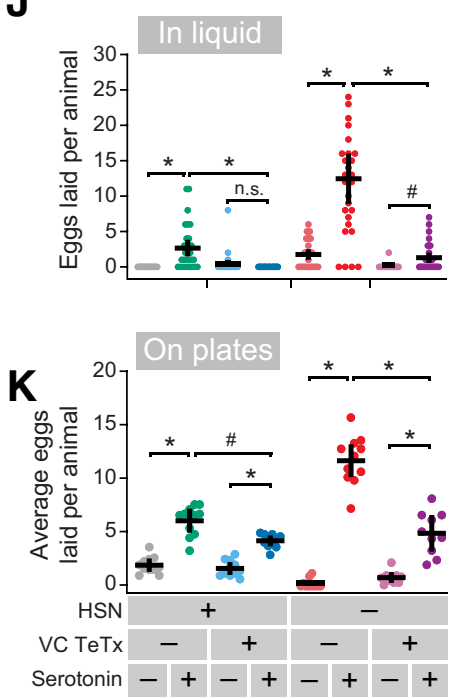

L

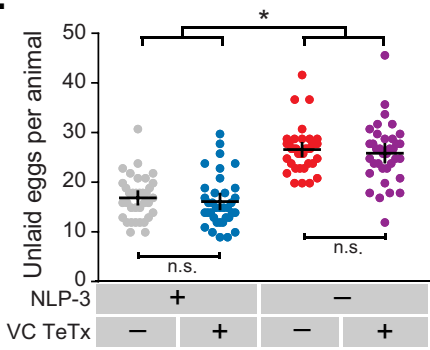

M

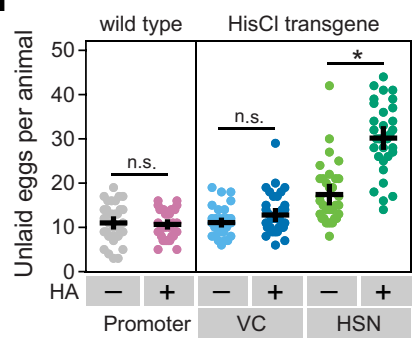

N

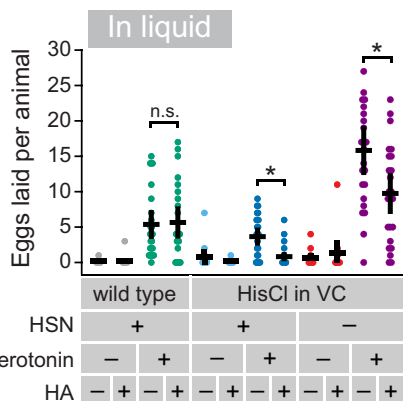

Figure 1. VC neurotransmission facilitates egg laying in response to serotonin. $\boldsymbol{A}$, Graphical representation of the C. elegans egg-laying circuit (modified from Collins et al., 2016). $\boldsymbol{B}$, Simplified circuit diagram showing the synapses and the primary neurotransmitters released between the HSN neurons, VC neurons, and vulval muscles. The cell-specific transgene expressions performed throughout this figure are indicated. $\mathbf{C}-\boldsymbol{F}$, Representative images of the $C$. elegans uterus showing unlaid eggs (arrowheads) in WT, transgenic animals expressing TeTx in the V(S, and $\mathrm{HSN}$-ablated egl-1 $(986 \mathrm{dm})$ mutant animals. G, Measurement of steady-state egg accumulation in animals from Figure $1\left(-F\left( \pm 95\right.\right.$ Cls for the mean). ${ }^{*} p<0.0001$. n.s. indicates $p>0.05$ (one-way ANOVA with Bonferroni's correction for multiple comparisons). $n \geq 17$. H, Scatterplot represents timing of first egg laid after the larval to adult molt in animals with blocked VC neurotransmission and ablated HSNs. ${ }^{*} p<0.0001$ (one-way ANOVA with Bonferroni's correction for multiple comparisons). $n \geq 17$. I, Blockage of VC synaptic transmission inhibits serotonininduced egg laying. Animals expressing TeTx in the VCs were placed into M9 buffer or M9 containing the indicated concentrations of serotonin and scored for number of eggs laid. ${ }^{*} p<0.0001$ (Kruskal-Wallis test with Dunn's correction for multiple comparisons). $n \geq 32$. J, Blockage of VC synaptic transmission inhibits serotonin-induced egg laying in HSN-ablated animals. HSN-ablated egl-1 $\left(986 \mathrm{dm}\right.$ ) mutant animals expressing TeTx in the VCs were placed in M9 buffer or M9 containing 18.5 mm serotonin. ${ }^{*} p \leq 0.0002$. ${ }^{*} p=0.0225$. n.s. indicates $p>0.05$ (Kruskal-Wallis test with Dunn's correction for multiple comparisons). $n \geq 22$. $\boldsymbol{K}$, Animals with inhibited VC neurotransmission lay fewer eggs compared with WT animals in response to 18.5 mм serotonin under otherwise normal culturing conditions on solid media agar plates. ${ }^{*} p<0.0001 .{ }^{*} p=0.0026$. n.s. indicates $p>0.05$ (one-way ANOVA with Bonferroni's correction for multiple comparisons). $n \geq 10$. L, Measurement of steady-state egg accumulation in animals expressing TeTx in the VCs in an $n / p-3($ tm3023) mutant background ( \pm 95 (ls for the mean). ${ }^{*} p<0.0001$. n.s. indicates $p>0.05$ (one-way ANOVA with Bonferroni's correction for multiple comparisons). $n \geq 35$. $\boldsymbol{M}$, Measurement of steady-state egg accumulation in animals expressing 
Table 1. Strain names and genotypes for all animals used in this study

\begin{tabular}{|c|c|c|c|}
\hline Strain & Genotype & Feature & Reference \\
\hline N2 & WT & Bristol WT strain & Brenner, 1974 \\
\hline CB190 & unc-54(e190) I & Myosin heavy chain null mutant & Brenner, 1974 \\
\hline LX1832 & lite-1(ce314) X lin-15(n765ts) X & $\begin{array}{l}\text { Blue light-resistant (optogenetics and } \mathrm{Ca}^{2+} \text { imaging), multivulva } \\
\text { (injection rescue marker) }\end{array}$ & Collins and Koelle, 2013 \\
\hline LX1918 & vsls164 lite-1(ce314) lin-15(n765ts) X & Vulval muscles expressing GCaMP5, mCherry & Collins et al., 2016 \\
\hline LX1960 & vsIs172; lite-1(ce314) lin-15(n765ts) X & VC GCaMP5, mCherry & Collins et al., 2016 \\
\hline LX1970 & wzls30 IV; vsls172; lite-1(ce314) lin-15(n765ts) X & HSN ChR2; VC GCaMP5, mCherry & Collins et al., 2016 \\
\hline MIA26 & egl-1(n986dm) V & No HSNs & Ravi et al., 2018a \\
\hline MIA116 & keyls21; lite-1(ce314) lin-15(n765ts) X & HSN HisCl & Ravi et al., 2018a \\
\hline MIA123 & egl-1(n986dm) V lite-1(ce314) lin-15(n765ts) X & No HSNs & Present study \\
\hline MIA125 & key/s23; lite-1(ce314) lin-15(n765ts) X & VC HisCl & Present study \\
\hline MIA144 & key/s33; lite-1(ce314) lin-15(n765ts) X & VC TeTx & Present study \\
\hline MIA146 & key/s46; lite-1(ce314) lin-15(n765ts) X & VC TeTx & Present study \\
\hline MIA221 & keyls3; vsls164; lite-1(ce314) lin-15(n765ts) X & VC ChR2; vulval muscle GCaMP5, mCherry & Present study \\
\hline MIA229 & key/s48; lite-1(ce314) lin-15(n765ts) X & Vulval muscle ChR2 & Present study \\
\hline MIA241 & keyls48; vsls172; lite-1(ce314) lin-15(n765ts) X & Vulval muscle ChR2; VC GCaMP5, mCherry & Present study \\
\hline MIA250 & keyls48; vsls164 lite-1(ce314) lin-15(n765ts) X & Vulval muscle ChR2, GCaMP5, mCherry & Present study \\
\hline MIA298 & key/s48; vs/s172; unc-54(e190) I; lite-1(ce314) lin-15(n765ts) X & $\begin{array}{l}\text { Vulval muscle ChR2; VC GCaMP5, mCherry; unc-54 myosin heavy } \\
\text { chain null mutant background }\end{array}$ & Present study \\
\hline MIA428 & nlp-3(tm3023); keyls33 & VC TeTx; nlp-3 null & Present study \\
\hline
\end{tabular}

VC ChR2. The allele keyIs 3 was used to express ChR2 in the VC neurons under a modified lin-11 promoter, as previously described (Collins et al., 2016).

HSN ChR2. The allele wzIs30 was used to express ChR2 in the HSN neurons under the egl-6 promoter, as previously described (Emtage et al., 2012; Collins et al., 2016).

\section{Calcium reporter transgenes}

Vulval muscle GCaMP5. Vulval muscle $\mathrm{Ca}^{2+}$ activity was visualized using the strain LX1918, which coexpresses GCaMP5 and mCherry in the vulval muscles from the unc-103e promoter (Collins et al., 2016). To analyze vulval muscle $\mathrm{Ca}^{2+}$ activity in animals where VC synaptic transmission was blocked with TeTx, LX1918 was crossed with MIA144 to generate MIA183 keyIs33; vsIs164 lite-1(ce314) lin-15(n765ts) X. To analyze vulval muscle $\mathrm{Ca}^{2+}$ activity in animals where the VC neurons could be optogenetically activated by ChR2, LX1918 was crossed with MIA3 (Collins et al., 2016) to generate MIA221 keyIs3; vsIs164 lite-1(ce314) lin15 (n765ts) X. To analyze vulval muscle $\mathrm{Ca}^{2+}$ activity in animals where the vulval muscles could be optogenetically activated by ChR2, LX1918 was crossed with MIA229 to generate MIA250 keyIs49; vsIs164 lite-1 (ce314) lin-15(n765ts) X.

VC neuron GCaMP5. VC neuron $\mathrm{Ca}^{2+}$ activity was visualized using the strain LX1960, which coexpresses GCaMP5 and mCherry in the VC neurons (Collins et al., 2016). To visualize VC activity during optogenetic stimulation of the HSNs, the strain LX1970 was used (Collins et al., 2016). To visualize VC activity after optogenetic stimulation of the vulval muscles, LX1960 was crossed with MIA229 to generate MIA241 vsIs172;

HisCls in either the VC or HSN neurons grown with or without histamine. ${ }^{*} p<0.0001$. n.s. indicates $p>0.05$ (one-way ANOVA with Bonferroni's correction for multiple comparisons). $n \geq 33$. N, Acute electrical silencing of the VCs blocks serotonin-induced egg laying. Animals expressing HisCl in the VCs in either a WT or HSN-ablated egl- $1(986 \mathrm{dm})$ mutant background were incubated with 0 or $4 \mathrm{~mm}$ histamine for $4 \mathrm{~h}$, placed into wells with M9 buffer with 0 or $18.5 \mathrm{~mm}$ serotonin, and the number of eggs laid after $1 \mathrm{~h}$ were counted. ${ }^{*} p<0.05$. n.s. indicates $p>0.05$ (Kruskal-Wallis test with Dunn's correction for multiple comparisons). $n \geq 12$ for control M9 and $n \geq 24$ for serotonin.
keyIs48 lite-1(ce314) lin-15(n765ts) X. To visualize VC activity after optogenetic stimulation of the vulval muscles when muscle contraction is impaired, CB190 unc-54(e190) I myosin heavy chain null mutants were crossed with LX1832 to generate MIA274 unc-54(e190) I; lite-1(ce314) lin-15(n765ts) X. MIA274 was then crossed with MIA241 to generate MIA298 keyIs48; vsIs172; unc-54(e190) I; lite-1(ce314) lin-15(n765ts) X.

HSN neuron GCaMP5. HSN neuron $\mathrm{Ca}^{2+}$ activity was visualized using the strain LX2004, which coexpresses GCaMP5 and mCherry in the HSN neurons (Collins et al., 2016). To visualize HSN Ca ${ }^{2+}$ after neurotransmission from the VC neurons is blocked by TeTx, LX2004 was crossed with MIA144 to generate MIA217 keyIs33; vsIs183 lite-1(ce314) lin-15(n765ts)X.

\section{Ratiometric $\mathrm{Ca}^{2+}$ imaging}

Ratiometric $\mathrm{Ca}^{2+}$ imaging of the vulval muscles and VC neurons in freely behaving animals was performed as previously described (Ravi et al., 2018b). Late L4 hermaphrodites were staged and then imaged $24 \mathrm{~h}$ later by being moved to an NGM agar chunk between two glass coverslips. Animals were recorded on a Carl Zeiss Axio Observer.Z1 inverted compound microscope with a $20 \times 0.8 \mathrm{NA}$ Apochromat objective. Brightfield recordings of behavior were done with infrared illumination using a FLIR Grasshopper 3 CMOS camera after $2 \times 2$ binning using FlyCap software. Colibri.2 $470 \mathrm{~nm}$ and $590 \mathrm{~nm}$ LEDs were used to coexcite GCaMP5 and mCherry fluorescence, which was captured at $20 \mathrm{~Hz}$ onto a Hamamatsu ORCA Flash-4.0V2 sCMOS camera after channel separation using a Gemini image splitter. For $\mathrm{Ca}^{2+}$ imaging of the vulval muscles, a lateral focal plane was used to capture the anterior and posterior vm 1 and vm 2 cells on one side of the animal. For $\mathrm{Ca}^{2+}$ imaging of the VC neurons, a lateral focal plane was used to capture the VC4 and VC5 cell bodies and their presynaptic termini around the vulva. Each animal was recorded until it entered an active egg-laying phase (up to 1 h), after which a 10 min segment centered around the onset of egg laying was extracted from the full recoding for analysis. Two-channel fluorescence (GCaMP5/mCherry) image sequences were processed and analyzed in Fiji (Schindelin et al., 2012), Volocity (PerkinElmer), and a custom script for MATLAB (The MathWorks) as previously described (Ravi et al., 2018b). 
Ratiometric $\mathrm{Ca}^{2+}$ imaging of the HSN neurons in freely behaving animals was performed as previously described (Ravi et al., 2018a). Late L4 hermaphrodites were staged and then imaged $24 \mathrm{~h}$ later by being moved to an NGM agar chunk and a glass coverslip being placed over. Animals were recorded on an inverted Leica Microsystems TCS SP5 confocal microscope with a $20 \times 0.7 \mathrm{NA}$ Apochromat objective; $488 \mathrm{~nm}$ and $561 \mathrm{~nm}$ laser lines were used to co-excite GCaMP5 and mCherry fluorescence, respectively.

\section{Electrical silencing using $\mathrm{His} \mathrm{Cl}$}

Acute electrical silencing with histamine was performed as previously described (Pokala et al., 2014; Ravi et al., 2018a). Animals were moved onto OP50-seeded NGM agar plates that contained either 0 or $10 \mathrm{~mm}$ histamine for $4 \mathrm{~h}$ before the experiment.

\section{Egg-laying behavior assays}

The steady-state accumulation of eggs in the uterus was determined as previously described (Koelle and Horvitz, 1996). Briefly, late L4 hermaphrodites were staged onto OP50-seeded plates and grown at $20^{\circ} \mathrm{C}$ for $30 \mathrm{~h}$ after which a single adult was placed into a $7 \mu \mathrm{ldrop}$ of $20 \%$ sodium hypochlorite (bleach) solution. The eggs, which are resistant to bleach, were then counted using a dissecting microscope. The timing of the first egg laid was assayed by staging a young adult (within $30 \mathrm{~min}$ of L4 to adult molt) animal onto an NGM agar plate with food and checking every following $30 \mathrm{~min}$ for the presence of an egg on the plate (Ravi et al., 2018a).

Egg laying in liquid in response to exogenous serotonin was performed as described previously (Trent et al., 1983; Banerjee et al., 2017). Late L4 hermaphrodites were staged onto OP50-seed plates and grown at $20^{\circ} \mathrm{C}$ for $24 \mathrm{~h}$. Adult animals were placed singly into either $100 \mu \mathrm{l} \mathrm{M} 9$ buffer only or M9 buffer containing $18.5 \mathrm{~mm}$ serotonin creatinine sulfate salt (Sigma Millipore) in a 96-well microtiter dish. The number of eggs laid by each animal after $1 \mathrm{~h}$ were then counted. Egg laying on plates in response to exogenous serotonin was performed on NGM agar infused with $18.5 \mathrm{~mm}$ serotonin creatine sulfate salt (Sigma Millipore). Three animals were placed on each NGM agar plate, and the number of eggs laid was counted after $1 \mathrm{~h}$ and divided by 3 to calculate an average-animal response per plate.

\section{Optogenetics}

Optogenetic experiments with ChR2 were performed using a Carl Zeiss Axio Observer.Z1 inverted compound microscope as previously described (Collins et al., 2016). ChR2 was excited in freely behaving animals using a $470 \mathrm{~nm}$ (blue) LED. Late L4 animals were staged onto NGM agar plates seeded with E. coli OP50 bacterial cultures containing either $0.4 \mathrm{~mm}$ all-trans retinal (ATR) or no ATR $24 \mathrm{~h}$ before the start of the experiment. Animals were then continuously illuminated with blue light for 3-5 $\mathrm{min}$, and the locomotion behavior and number of egg-laying events were recorded. For experiments combining optogenetics with ratiometric $\mathrm{Ca}^{2+}$ imaging, the blue light would excite both the ChR2 and GCaMP5 fluorescence simultaneously. Blue light intensity was chosen based on both optimal settings to observe robust ChR2 activation and GCaMP5 fluorescence. Animals were excluded from a dataset if they entered an active egg-laying state before the onset of blue light stimulation (one animal in total across all experiments).

\section{Experimental design and statistical analyses}

Sample sizes for behavioral assays and $\mathrm{Ca}^{2+}$ imaging experiments followed previous studies (Collins et al., 2016; Banerjee et al., 2017; Ravi et al., 2018a). A minimum of 10 animals per genotype per condition were measured across all experiments. One animal was excluded from the analysis of VC ChR2; vulval muscles GCaMP5 experiment because it entered an egg-laying active state before the onset of blue light stimulation. No other data or animals were excluded. No explicit power analysis was performed before the study. All data were analyzed using GraphPad Prism 8. Steady-state egg accumulation and timing of first egg laid assays were compared using a one-way ANOVA with Bonferroni's correction for multiple comparisons. Serotonin-induced egg laying assays were compared using either a Kruskal-Wallis test with Dunn's correction for multiple comparisons (in buffer with individual responses) or a one-way ANOVA with Bonferroni's correction for multiple comparisons (on plates with averaged responses). Intertransient intervals of $\mathrm{Ca}^{2+}$ transients compared between active and inactive egg-laying behavior states were pooled together across all animals and analyzed using either a Kolmogorov-Smirnov test or Kruskal-Wallis test with Dunn's correction for multiple comparisons. Per animal, rates of failed egg-laying events were analyzed using a Mann-Whitney test. $\mathrm{Ca}^{2+}$ transient peak amplitude, intertransient interval, or transient widths were first averaged across each animal, and then these averages were compared across animals using either a Student's $t$ test or a one-way ANOVA with Bonferroni's correction for multiple comparisons. The number of animals and instances of analyzed behavior events along with exact $p$ values resulting from defined statistical tests are reported within each figure legend.

\section{Results}

The VCs are a group of 6 hermaphrodite-specific, cholinergic motor neurons spaced out along the ventral cord that make synapses onto the HSNs, vm2 vulval muscles, body wall muscles, and various motor neurons involved in locomotion (Fig. 1A) (White et al., 1986; Cook et al., 2019). VC4 and VC5, in particular, are the most proximal to the vulva and make extensive synapses onto the vm2 vulval muscles (White et al., 1986; Cook et al., 2019). Egg-laying events are always accompanied by a VC $\mathrm{Ca}^{2+}$ transient, but not all $\mathrm{VC} \mathrm{Ca}{ }^{2+}$ transients coincide with egg release (Collins et al., 2016). ACh released from VCs has been suggested to act through nAChRs, including UNC-29 on the vulval muscles to drive contraction (Kim et al., 2001; Schafer, 2006), as well as through the muscarinic ACh receptor GAR-2 on the HSNs to inhibit egg laying (Bany et al., 2003; Fernandez et al., 2020). VC signaling has also been suggested to slow animal locomotion speed around egg-laying events (Collins et al., 2016), likely through the synapses it makes onto the body wall muscles and locomotion motor neurons (White et al., 1986; Cook et al., 2019). In all, VC signaling appears to be complex and function through multiple pathways to regulate egg-laying circuit activity and behavior.

The vulval muscles are comprised of four vm1-type and four vm2-type muscle cells, which are all electrically coupled but receive distinct synaptic input (White et al., 1986). The vm2 vulval muscles are innervated by the HSN and VC neurons (White et al., 1986; Cook et al., 2019). The vm1s receive cholinergic input from single VA and VB motor neurons, which are part of the locomotion circuit (White et al., 1986; Zhen and Samuel, 2015). Egg laying occurs when all 8 vulval muscle cells are active and pull the vulva open to expel an egg (Zhang et al., 2008). Serotonergic input from the HSNs helps to excite the vm2 vulval muscles and coordinate their activity during egg laying (Shyn et al., 2003; Li et al., 2013; Brewer et al., 2019). The vm1 vulval muscles show rhythmic activity both during and outside of egg laying, suggesting that they are either intrinsically active or excited by VA and VB synaptic inputs (Collins and Koelle, 2013; Li et al., 2013; Collins et al., 2016). Vulval muscle $\mathrm{Ca}^{2+}$ transients start in the vm1 muscle cells and propagate into the vm 2 muscles during egg-laying events (Collins and Koelle, 2013; Brewer et al., 2019), suggesting that vm1 excitation provides a trigger for full vulval muscle contraction and egg laying.

The pair of hermaphrodite-specific HSN neurons make synapses onto both the VCs and the vm2 vulval muscles (White et al., 1986; Cook et al., 2019). Serotonin and NLP-3 neuropeptides released by the HSNs have been shown to be critical for normal egg-laying behavior to occur (Waggoner et al., 1998; Brewer et al., 2019). Consistent with the role of HSNs initiating egg-laying 
behavior, exogenous serotonin potently increases the $\mathrm{Ca}^{2+}$ activity of the vulval muscles and VC neurons (Shyn et al., 2003; Zhang et al., 2008). Additionally, laser ablation experiments have indicated that loss of the VC neurons disrupts the induction of egg laying in response to serotonin (Waggoner et al., 1998; Shyn et al., 2003). Thus, the role of the VC neurons in the egg-laying circuit and behavior may be closely associated with serotoninergic signaling from the HSNs.

\section{The VC neurons promote egg laying in response to serotonin}

To test directly how loss of synaptic transmission from the VC motor neurons affects egg-laying circuit activity and behavior (Fig. $1 B)$, we used a modified lin-11 promoter/ enhancer (Bany et al., 2003) to drive transgenic expression of TeTx (Jose et al., 2007), which blocks both neurotransmitter and neuropeptide release (Whim et al., 1997) from the six VC neurons. Expression of TeTx in the VC neurons did not cause any gross defects in steady-state egg accumulation compared with nontransgenic control animals, indicating that the VC neurons are not strictly required for egg laying (compare Fig. 1C,D, quantified in Fig. $1 G$ ). In contrast, egl-1(n986dm) mutant animals in which the HSNs undergo apoptosis (Trent et al., 1983) showed a dramatic impairment of egg laying, accumulating significantly more embryos (Fig. 1E,G). Previous studies indicated that laser ablation of both the HSNs and VCs caused additive defects in egg laying (Waggoner et al., 1998). However, transgenic expression of TeTx in the VCs in HSN-deficient egl-1 (n986dm) mutant animals did not significantly enhance their defects in egg laying (Fig. $1 F, G$ ). We have previously shown that WT animals lay their first egg at $\sim 7 \mathrm{~h}$ after the L4-adult molt, a time when the VCs show their first activity (Ravi et al., 2018a). Animals with inhibited VC neurotransmission showed no significant change in the onset of egg laying compared with nontransgenic control animals (Fig. $1 H$ ). In contrast, the onset of egg laying in egl-1 ( $n 968 d m)$ mutant animals lacking HSNs is significantly delayed, occurring $\sim 18 \mathrm{~h}$ after the L4-adult molt (Fig. 1H). Expression of TeTx in the VCs in HSN-deficient animals did not enhance the delay in egg laying significantly (Fig. $1 H$ ). These results together show that transmitter-mediated signaling from the VCs is not required for egg-laying behavior to occur under normal culturing conditions.

We next used a drug-treatment approach to explore possible functions of the VC neurons that may not be apparent in animals under standard laboratory conditions. Serotonin potently stimulates egg laying, even in conditions where egg laying is normally
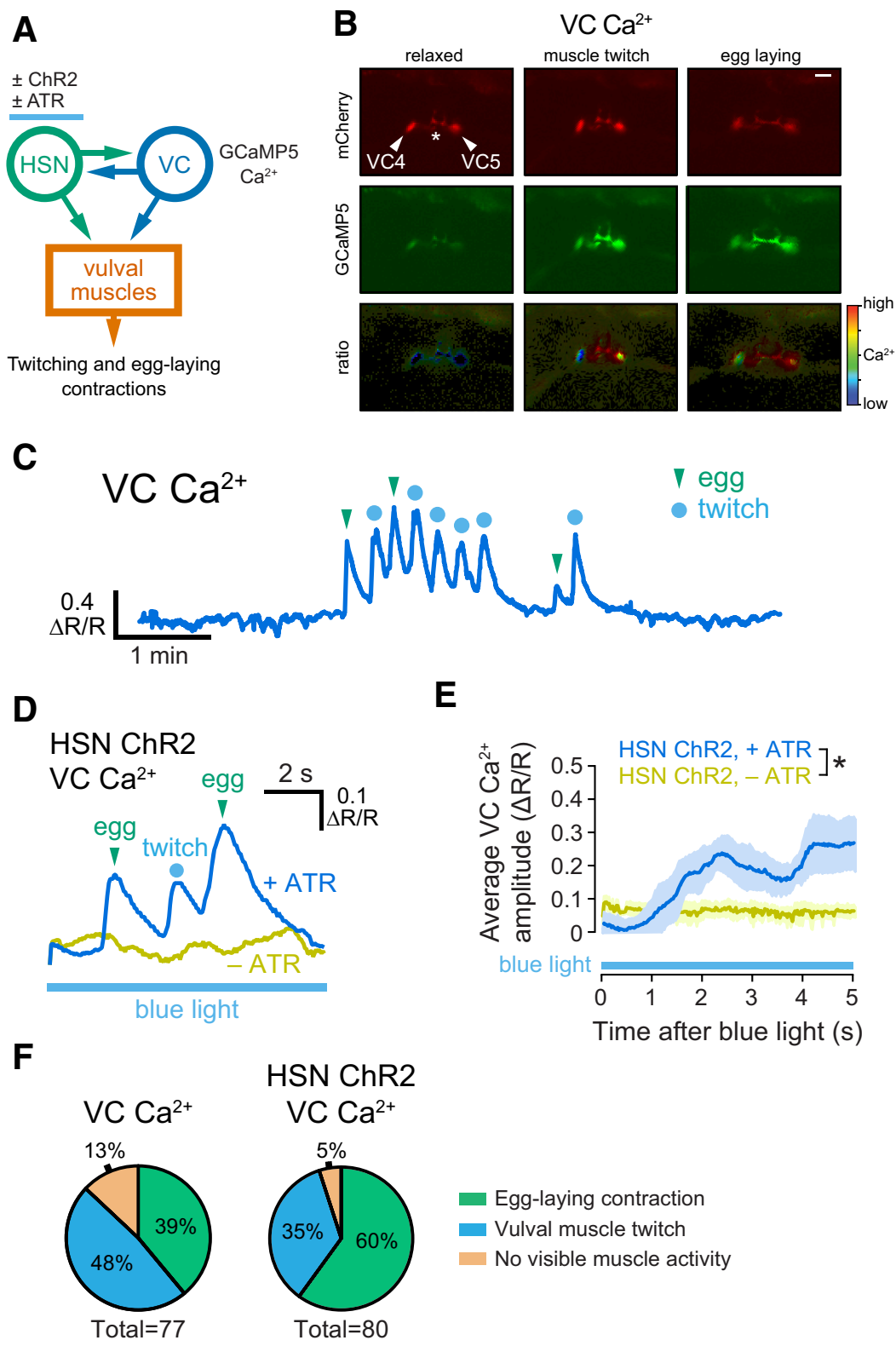

Figure 2. The VC neurons are active during both weak vulval muscle twitching and strong egg-laying contractions. $\boldsymbol{A}$, Diagram of the circuit and experimental manipulations. GCaMP5 was expressed in the VC neurons to record $\mathrm{Ca}^{2+}$ activity, and ChR2 was expressed in HSNs to provide optogenetic stimulation of egg laying. $\boldsymbol{B}$, Representative still images of VC mCherry, GCaMP5, and GCaMP5/mCherry ratio $(\triangle R / R)$ during inactive and active egg-laying behavior states. *Vulva. Scale bar, $20 \mu \mathrm{m}$. C, Representative trace of VC GCaMP5/mCherry $\mathrm{Ca}^{2+}$ ratio in freely behaving, WT animals during an egg-laying active state. $\boldsymbol{D}$, Representative trace of VC GCaMP5/mCherry $\mathrm{Ca}^{2+}$ ratio after optogenetic stimulation of ChR2 in the HSN neurons in animals grown in the absence (-ATR, top) and presence ( + ATR, bottom) during $10 \mathrm{~s}$ of continuous blue light exposure. $\boldsymbol{E}$, Average vulval muscle $\mathrm{Ca}^{2+}$ levels (mean $\pm 95 \% \mathrm{Cls}$ ). ${ }^{*} p<0.0001$ (Student's $t$ test). $n=10 . \boldsymbol{F}, \mathrm{Graph}$ represents vulval muscle contractile activity for each $\mathrm{VC} \mathrm{Ca^{2+ }}$ transient during endogenous egg-laying active states (left) or in response to HSN optogenetic stimulation (right).

inhibited, such as in liquid M9 buffer (Trent et al., 1983). As expected, serotonin promotes egg laying in M9 buffer in WT animals (Fig. 1I). We found that transgenic animals expressing TeTx in the VCs failed to lay eggs in response to exogenous serotonin in M9 buffer across varying serotonin concentrations (Fig. 1I). Going forward, we chose to conduct experiments at a single serotonin concentration of $18.5 \mathrm{~mm}$. HSN-deficient egl-1 $(n 986 \mathrm{dm})$ mutant animals are egg-laying defective under normal conditions but will still lay eggs in response to exogenous serotonin (Schafer et al., 1996), and this response was 
suppressed in animals lacking VC neurotransmission at 18.5 mu serotonin (Fig. 1J). This resistance to exogenous serotonin in VC neurotransmission-inhibited animals was not unique to M9 buffer, as transgenic animals placed on serotonin-infused agar also showed a reduced egg-laying response (Fig. $1 \mathrm{~K}$ ). These results show that VC neurotransmitter release serves an important role for egg laying in response to exogenous serotonin.

Despite the VCs being important for egg laying in response to exogenous serotonin (Fig. $1 I-K$ ), animals with blocked VC neurotransmission are still able to lay eggs at a normal rate (Fig. $1 G$ ). One potential explanation for this is that NLP-3 neuropeptide signaling is able to compensate for the loss of the VC-mediated serotonergic signaling pathway (Brewer et al., 2019). Based on this model, blocking VC neurotransmission in an $n l p-3$ mutant background might phenocopy animals lacking all neurotransmitter release from the HSNs, as seen in the egl-1(n986dm) mutant. However, we found no significant increase in egg accumulation when blocking VC neurotransmission in an $n l p-3$ mutant background (Fig. $1 L$ ). This suggests that, under standard C. elegans culture conditions, serotonin is able to signal through VC-independent pathway to retain a normal rate of egg laying, likely by acting on the vulval muscles directly (Hapiak et al., 2009).

It was possible that the observed defects in serotonin response caused by inhibiting VC neurotransmission could be because of impaired circuit development and/or by compensatory changes in circuit activity. Expression from the VC-specific promoter used to drive TeTx begins in the L4 stage as the egg-laying circuit is completing development, well before the onset of egg-laying behavior (Ravi et al., 2018a). To silence the VCs acutely after circuit development is complete, we transgenically expressed HisCls and treated the animals with exogenous histamine (Pokala et al., 2014; Ravi et al., 2018a). Histamine silencing of the VCs caused no gross changes in steady-state egg accumulation (Fig. 1M), confirming the results with TeTx that VC neurotransmission is not required for egg laying. However, acute histamine silencing of the VCs reduced egg laying in response to serotonin in both WT and HSN-deficient egl-1(n986dm) mutant animals (Fig. 1N), consistent with the results observed when blocking VC neurotransmission with TeTx. Together, these results show that both VC neuron activity and synaptic transmission are dispensable for egg laying under normal growth conditions, but the VCs do facilitate egg laying in response to exogenous serotonin.

\section{VC Ca ${ }^{2+}$ activity is coincident with vulval muscle activity and egg laying}

The VC neurons show rhythmic $\mathrm{Ca}^{2+}$ activity during the egglaying active state (Collins et al., 2016). However, only approximately one-third of $\mathrm{VC} \mathrm{Ca}^{2+}$ transients were temporally coincident with vulval muscle contractions that resulted in egg release (Collins et al., 2016), raising questions about the function of the $\mathrm{VC} \mathrm{Ca}{ }^{2+}$ transients that do not coincide with egg laying. To understand the function of $\mathrm{VC} \mathrm{Ca}^{2+}$ activity, we expressed GCaMP5 in the VC neurons to measure $\mathrm{Ca}^{2+}$ dynamics in the $\mathrm{VC}$ cell bodies and processes most proximal to the vulva (VC4 and VC5; Fig. $2 A, B$ ) while simultaneously observing vulval opening and egg-laying behavior in a separate brightfield channel, as described previously (Ravi et al., 2018b). Since C. elegans neurons generally exhibit graded potentials, measurements of $\mathrm{VC} \mathrm{Ca}{ }^{2+}$ near synapses can be expected to correspond closely with the degree of neurotransmitter and neuropeptide release (Q. Liu et al., 2009; P. Liu et al., 2013). As expected, we found that egg-

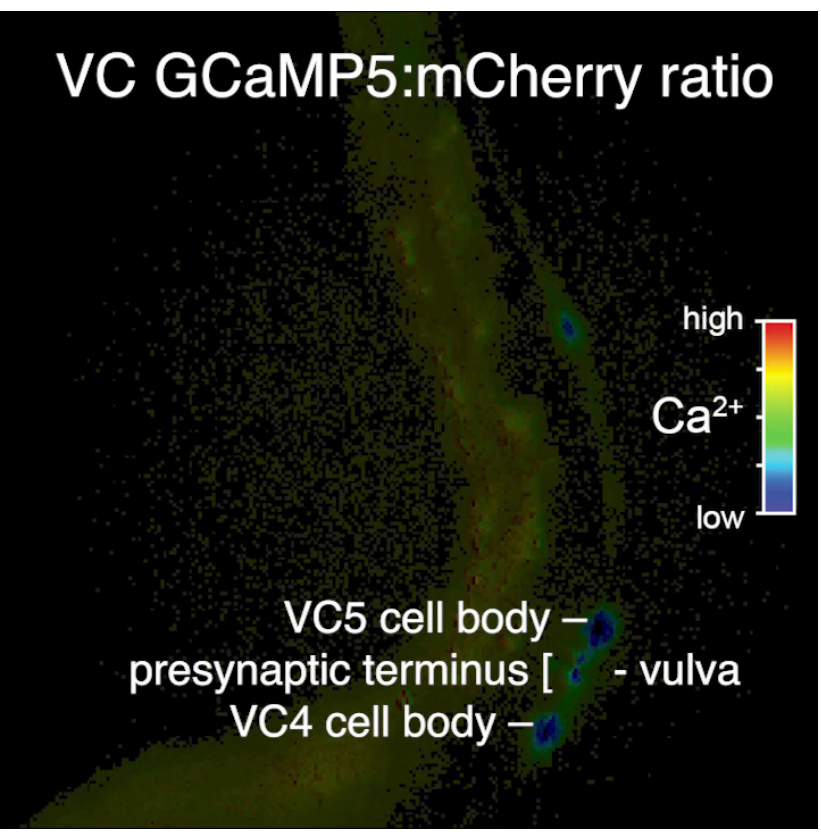

Movie 1. GCaMP5/mCherry ratio recording of $\mathrm{VC} \mathrm{Ca}^{2+}$ activity during the egg-laying active state. Ratio of GCaMP5 and mCherry fluorescence in the VC neurons mapped onto a false color spectrum ranging from blue (low $\left.\mathrm{Ca}^{2+}\right)$ to red (high $\left.\mathrm{Ca}^{2+}\right)$. The VC neurons show high $\mathrm{Ca}^{2+}$ activity and physical displacement as the vulval muscles contract and an egg passes through the vulva to be laid. The $\mathrm{Ca}^{2+}$ activity then returns to a low level until a vulval muscle twitch occurs (0:09) followed by another egg-laying event. [View online]

laying events were always accompanied by a $\mathrm{VC} \mathrm{Ca}^{2+}$ transient, but not every $\mathrm{VC} \mathrm{Ca}^{2+}$ transient resulted in an egg-laying event (Fig. 2C; Movie 1). These remaining $\mathrm{VC} \mathrm{Ca}^{2+}$ transients were almost always observed with weak muscle contraction and partial opening of the vulva, termed a vulval muscle "twitch," which are primarily mediated by the vm1 vulval muscles (Fig. 2C,F) (Collins and Koelle, 2013). As quantified in Figure 2F, we found that $48 \%$ of $\mathrm{VC} \mathrm{Ca}^{2+}$ transients were associated with vulval muscle twitch contractions, and $39 \%$ of $\mathrm{VC} \mathrm{Ca}^{2+}$ transients were associated with strong vulval muscle contractions that support egg release, which are the result of simultaneous vm1 and vm2 vulval muscle contraction (Collins and Koelle, 2013). To determine whether presynaptic HSN activity was sufficient to drive VC and vulval muscle activity downstream, we optogenetically stimulated HSNs transgenically expressing ChR2 and simultaneously recorded $\mathrm{VC} \mathrm{Ca}{ }^{2+}$ activity and vulval muscle contractility (Fig. 2A). As previously reported, optogenetic HSN stimulation was sufficient to induce an active egg-laying behavior state (Collins et al., 2016). We found that optogenetic stimulation of $\mathrm{HSN}$ drove a robust increase in $\mathrm{VC} \mathrm{Ca}{ }^{2+}$ transients that were associated with egg-laying events, but we still observed $\mathrm{VC} \mathrm{Ca}{ }^{2+}$ transients during the weaker vulval muscle twitching contractions (Fig. 2D). HSN optogenetic stimulation rapidly elevated average $\mathrm{Ca}^{2+}$ levels in the VCs, within $5 \mathrm{~s}$ after blue light exposure (Fig. 2E). The light-dependent increase in $\mathrm{VC} \mathrm{Ca}^{2+}$ activity and vulval muscle contractions were not observed in animals grown without the essential cofactor, ATR (Fig. 2D,E). During optogenetic HSN activation, $60 \%$ of $\mathrm{VC} \mathrm{Ca}^{2+}$ transients were associated with egg-laying events, whereas only $35 \%$ were associated with vulval muscle twitches, a significant difference from control animals not subjected to HSN optogenetic stimulation that we attribute to an increase in egg-laying frequency (Fig. $2 F$ ). Because $\mathrm{VC} \mathrm{Ca}{ }^{2+}$ activity rises at the same time as vulval opening and before egg release, these results are consistent with either a model 
A

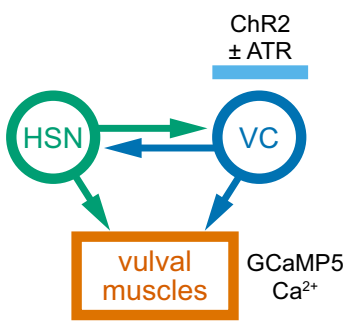

C

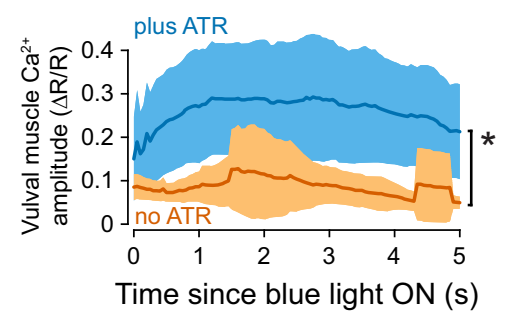

B

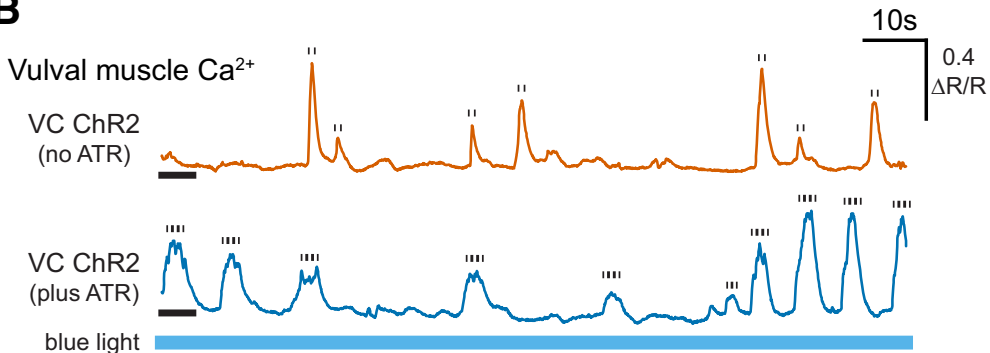

D

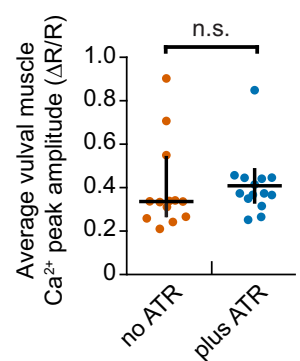

E

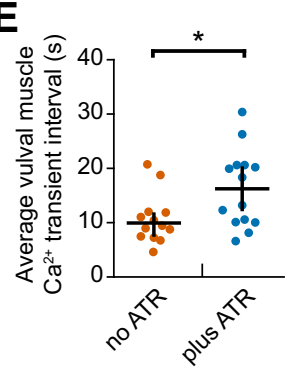

$\mathbf{F}$

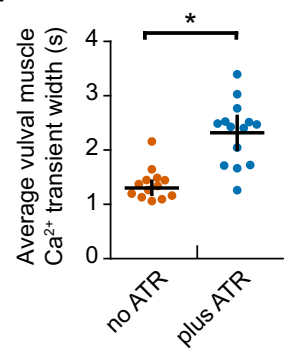

Figure 3. Optogenetic VC activation induces and sustains vulval muscle $\mathrm{Ca}^{2+}$ activity. $\boldsymbol{A}$, Diagram of circuit and experiment. ChR2 was expressed in the VC neurons, and GCaMP5 was expressed in the vulval muscles. $\boldsymbol{B}$, Representative traces of vulval muscle $\mathrm{GCaMP5} / \mathrm{mCherry} \mathrm{Ca}^{2+}$ ratio $(\Delta \mathrm{R} / \mathrm{R})$ in animals grown in the absence $(-\mathrm{ATR}$, top) or presence (+ATR, bottom) in response to $5 \mathrm{~min}$ of continuous blue light exposure. Tick marks represent duration at half-maximal amplitude of each measured $\mathrm{Ca}^{2+}$ transient. Black bar represents first $5 \mathrm{~s}$ following blue light exposure. C, Averaged vulval muscle $\mathrm{Ca}^{2+}$ transient activity (shaded region represents $\pm 95 \% \mathrm{Cls}$ ) during the first $5 \mathrm{~s}$ of optogenetic activation of the VC neurons in -ATR (control; blue) and +ATR conditions (orange). ${ }^{*} p=0.003$ (Student's $t$ test). $n \geq 13$ animals. D, Scatterplot represents the average peak amplitude of vulval muscle Ca ${ }^{2+}$ transients ( $\pm 95 \%$ Cls) per animal in response to VC optogenetic stimulation during $5 \mathrm{~min}$ of continuous blue light. n.s. indicates $p>0.05$ (Student's $t$ test). $n \geq 13$ animals. $E$, Scatterplot represents the average time between vulval muscle $\mathrm{Ca}^{2+}$ transients $\left( \pm 95 \% \mathrm{Cls}\right.$ ) per animal during VC optogenetic stimulation during 5 min of continuous blue light ${ }^{*} p=0.0236$ (Student's $t$ test). $n \geq 13$ animals. $\boldsymbol{F}$, Scatterplot represents the average vulval muscle $\mathrm{Ca}^{2+}$ transient width ( $\pm 95 \%$ (ls) per animal during VC optogenetic stimulation during 5 min of continuous blue light. ${ }^{*} p<0.0001$ (Student's $t$ test). $n \geq 13$ animals. Error bars indicate $95 \%$ Cls for the mean.

where the VCs promote vulval muscle contractility, or a model where the VCs are activated in response to downstream vulval muscle contraction.

\section{The VC neurons promote vulval muscle activity and contraction}

The VC motor neurons synapse onto the vulval and body wall muscles where they are thought to release ACh to drive contraction (White et al., 1986; Duerr et al., 2008; Zhang et al., 2008). While VC activity and neurotransmission are not required for egg laying (Fig. 1G,K) (Waggoner et al., 1998), the VCs may still release ACh to regulate vulval muscle contractility. To test whether the VCs can excite the vulval muscles directly, we expressed $\mathrm{ChR} 2$ in the VC neurons and performed ratiometric $\mathrm{Ca}^{2+}$ imaging in the vulval muscles after exposure to blue light (Fig. 3A; Movie 2). Optogenetic stimulation of the VCs led to an acute induction of vulval muscle $\mathrm{Ca}^{2+}$ activity within $5 \mathrm{~s}$ but was unable to drive full vulval muscle contractions and egg release (Fig. 3B,C). However, average vulval muscle $\mathrm{Ca}^{2+}$ transient amplitude after optogenetic stimulation was not significantly higher through the duration of the recording (Fig. 3D). Vulval muscle $\mathrm{Ca}^{2+}$ transient frequency was reduced, which may result from the increased duration of each individual transient (Fig. 3E,F). This result shows that $\mathrm{VC}$ activity alone is not able to maximally excite the vulval muscles to the point of egg laying, but that VC activity is excitatory and can sustain ongoing vulval muscle $\mathrm{Ca}^{2+}$ activity. We find these results consistent with a model where serotonin and NLP-3 neuropeptides released from the HSNs signal to enhance the excitability and contractility of the vulval muscles for egg laying (Brewer et al., 2019), whereas the ACh released from the VCs prolong the contractile phase to facilitate egg release.

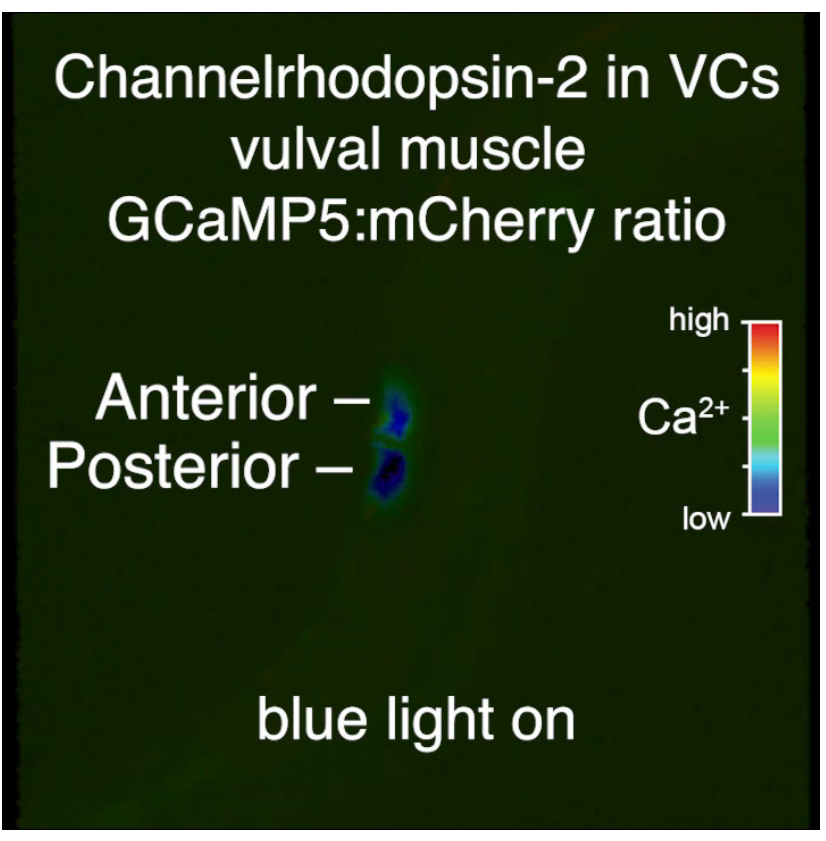

Movie 2. GCaMP5/mCherry ratio recording of vulval muscle $\mathrm{Ca}^{2+}$ in response to optogenetic stimulation of the VCs. Ratio of GCaMP5 and mCherry fluorescence in the vulval muscles mapped onto a false color spectrum ranging from blue (low $\mathrm{Ca}^{2+}$ ) to red (high $\left.\mathrm{Ca}^{2+}\right)$. Optogenetic activation of the VC neurons induces vulval muscle $\mathrm{Ca}^{2+}$ activity and twitches, but not egg laying. [View online]

\section{The VCs facilitate successful vulval opening during egg} laying

Loss of VC activity or synaptic transmission caused a specific defect in serotonin-induced egg laying, suggesting that the VCs 
A

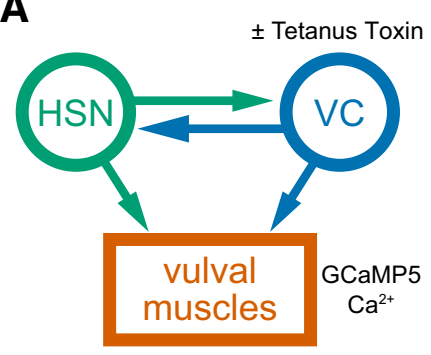

C

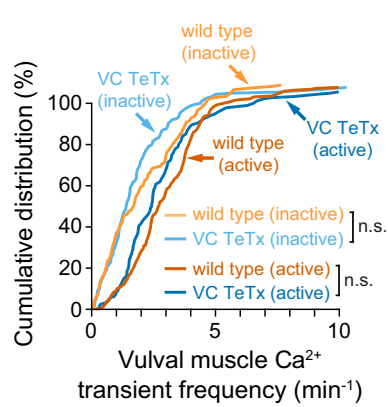

B

Bulval muscle $\mathrm{Ca}^{2+}$
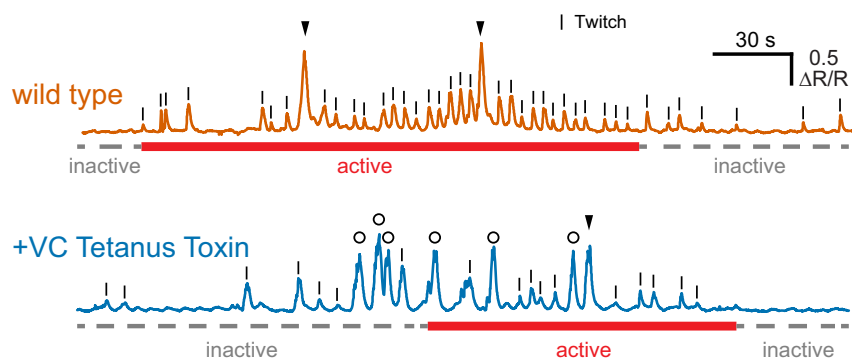

D

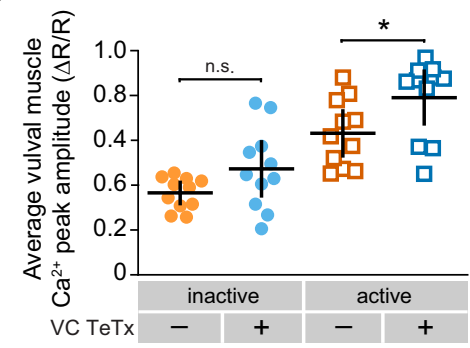

$\mathbf{E}$

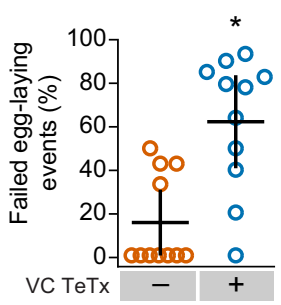

Figure 4. Blocking VC neurotransmission reduces the success rate of egg laying. $\boldsymbol{A}$, Diagram of circuit and experiment. TeTx was expressed in the VC neurons to block their neurotransmitter release, and GCaMP5 was expressed in the vulval muscles to record $\mathrm{Ca}^{2+}$ activity. $\boldsymbol{B}$, Representative traces of vulval muscle $\mathrm{GCaMP5} / \mathrm{mCherry} \mathrm{Ca}^{2+}$ ratio $(\Delta R / R)$ in WT (top) and TeTx-expressing transgenic animals (bottom). Arrowheads indicate successful egg-laying events. Open circles represent strong ( $>1.0 \Delta R / R)$ vulval muscle $\mathrm{Ca}^{2+}$ transients of "failed egg-laying events." Egg-laying behavior state (inactive or active) duration is indicated below each trace. $C$, Cumulative distribution plots of all vulval muscle $\mathrm{Ca}^{2+}$ transients across WT and transgenic animals expressing TeTx in the VCs. Transients were parsed into egg-laying active and inactive phases. n.s. indicates $p>0.05$ (Kruskal-Wallis test with Dunn's correction for multiple comparisons). $n \geq 200$ transients from 11 animals. $\boldsymbol{D}$, Average vulval muscle $\mathrm{Ca}^{2+}$ transient peak amplitudes per animal during the inactive and active egg-laying phase. ${ }^{*} p=0.0336$ (one-way ANOVA with Bonferroni's correction for multiple comparisons). $n=11$ animals. $\boldsymbol{E}$, Percentage of failed egg-laying events in WT and transgenic animals expressing TeTx in the VCs. ${ }^{*} p=0.0014$ (Mann-Whitney test). $n=11$ animals. Error bars indicate $95 \%$ Cls for the mean.

\section{Tetanus Toxin in the VCs, Vulval muscle GCaMP5:mCherry ratio}

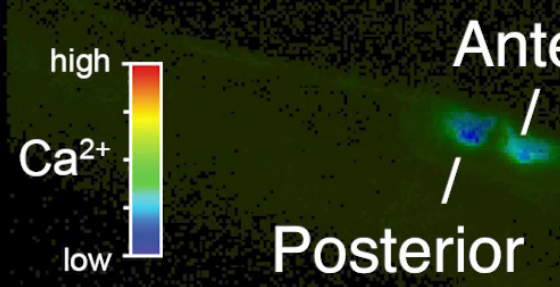

Movie 3. GCaMP5/mCherry ratio recording of vulval muscle $\mathrm{Ca}^{2+}$ during the egg-laying active state when synaptic transmission from the VC neurons is blocked by TeTx. Ratio of GCaMP5 and mCherry fluorescence in the vulval muscles mapped onto a false color spectrum ranging from blue (low $\mathrm{Ca}^{2+}$ ) to red (high $\mathrm{Ca}^{2+}$ ). The vulval muscles still exhibit twitches and egg-laying events but will also frequently fail to lay eggs in response to high $\mathrm{Ca}^{2+}$ activity, termed "failed egg-laying events." [View online]

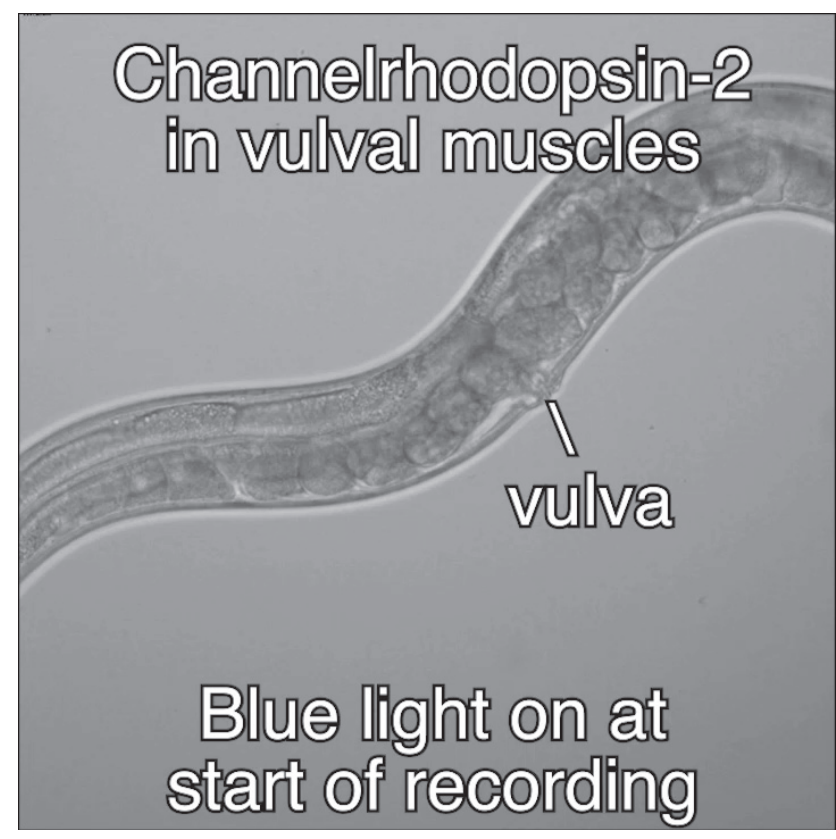

Movie 4. Brightfield recording of vulval opening and egg laying in response to optogenetic stimulation of the vulval muscles. Brightfield recording of egg laying in response to optogenetic vulval muscle activation. Optogenetic activation of the vulval muscles induces tetanic vulval muscle contraction, but vulval opening and egg release remain phased with the body bends of locomotion. [View online] 
A

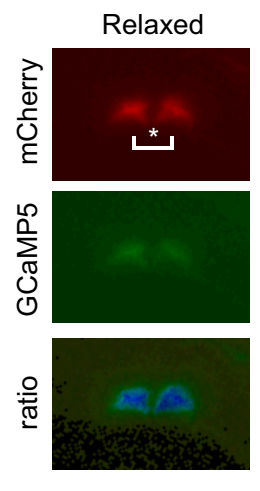

B

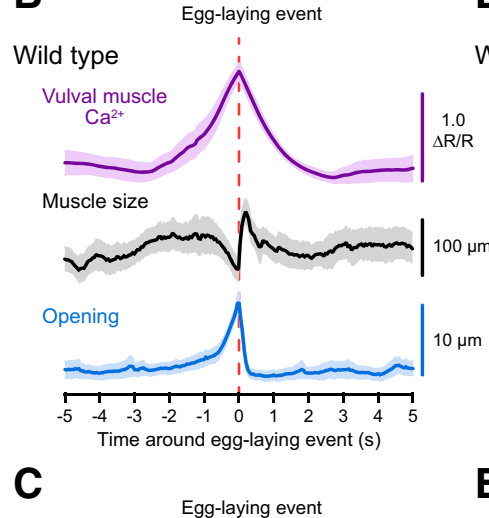

VC Tetanus Toxin
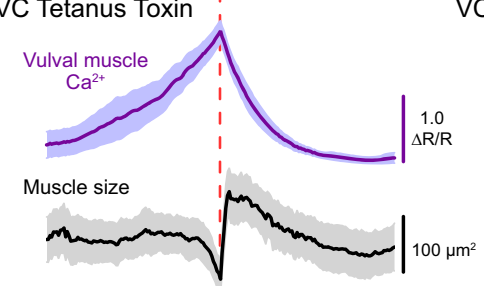

E

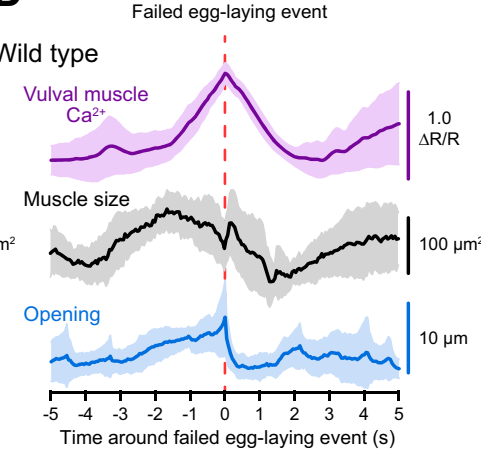

E

Failed egg-laying even

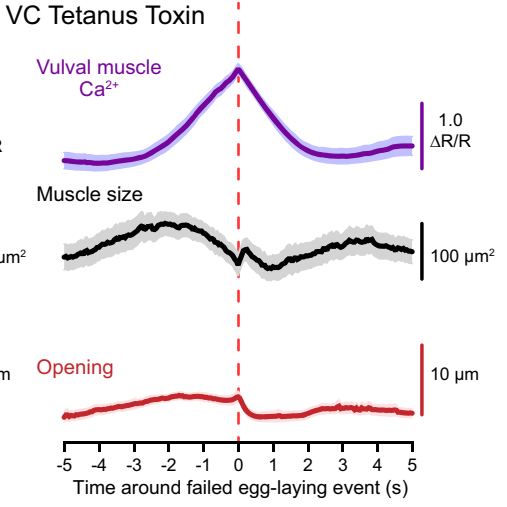

$\mathbf{F}$

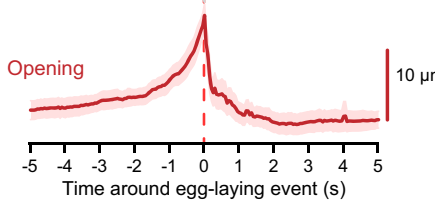

G

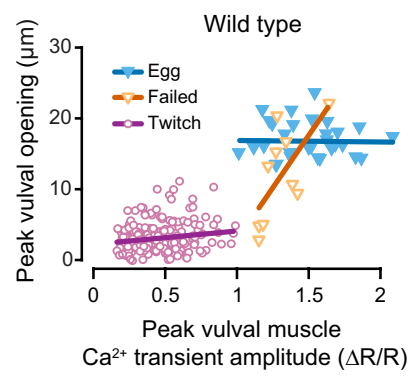

$\mathrm{Ca}^{2+}$ transient amplitude $(\Delta \mathrm{R} / \mathrm{R})$

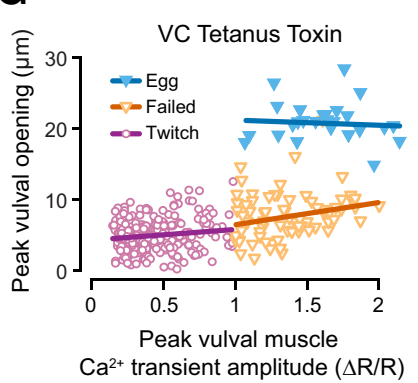

Figure 5. Blocking VC neurotransmission decouples vulval muscle $\mathrm{Ca}^{2+}$ activity and egg laying. $\boldsymbol{A}$, Vulval muscle sizes and distances were quantified by measuring the changes in area and centroid of the anterior and posterior muscle groups in mCherry channel micrographs during GCaMP5/mCherry ratiometric imaging. Shown are still images of the vulval muscles during representative muscle states of relaxation, twitching, egg laying, and failed egg-laying events. Brackets represent the distance between the centroid of vulval muscle halves. Arrowheads indicate high $\mathrm{Ca}^{2+}$ activity. *Vulva. Scale bar, $20 \mu \mathrm{m}$. $\boldsymbol{B}-\boldsymbol{D}$, Mean traces ( $\pm 95 \mathrm{Cls}$ ) of vulval muscle Ca ${ }^{2+}$ (GCaMP5/mCherry ratio), vulval muscle area $\left(\mu \mathrm{m}^{2}\right)$, and vulval muscle centroid distance $(\mu \mathrm{m})$ during successful $(\boldsymbol{B}, \boldsymbol{C} ; n \geq 26$ from 11 animals) and failed egg-laying events $(\boldsymbol{D}, \boldsymbol{E} ; n \geq 10$ from 11 animals) in WT $(\boldsymbol{B}, \boldsymbol{D})$ and transgenic animals expressing TeTx in the VC neurons $(C, E) . F, G$, Scatterplot represents peak vulval muscle $\mathrm{Ca}^{2+}$ amplitude in relation to the corresponding vulval

are required for proper vulval muscle $\mathrm{Ca}^{2+}$ activity and/or contractility. We recorded vulval muscle $\mathrm{Ca}^{2+}$ activity in freely behaving animals transgenically expressing TeTx in the VCs (Fig. $4 A$; Movie 3). Vulval muscle $\mathrm{Ca}^{2+}$ activity in WT animals is characterized by low activity during the $\sim 20 \mathrm{~min}$ egg-laying inactive state, and periods of high activity during the $\sim 2 \mathrm{~min}$ egg-laying active state (Fig. 4B) (Waggoner et al., 1998; Collins et al., 2016). Expression of TeTx in the VCs did not significantly affect the overall frequency or amplitude of vulval muscle $\mathrm{Ca}^{2+}$ transients during egg-laying inactive states compared with WT control animals (Fig. $4 C, D)$. However, inhibition of VC neurotransmission led to larger-amplitude $\mathrm{Ca}^{2+}$ transients during the active phase (Fig. $4 D$ ). Since previous work suggested the VCs release ACh that inhibits egg laying (Bany et al., 2003), the simplest explanation for this phenotype would be that the VCs normally inhibit vulval muscle $\mathrm{Ca}^{2+}$ activity. However, our results show optogenetic activation of the VCs increased vulval muscle $\mathrm{Ca}^{2+}$ transient duration with minimal effect on amplitude (Fig. 3C,D,F). Further inspection of vulval muscle $\mathrm{Ca}^{2+}$ traces of individual animals showed that transgenically expressing $\mathrm{TeTx}$ in the VCs had large vulval muscle $\mathrm{Ca}^{2+}$ transients of amplitude similar to egg-laying $\mathrm{Ca}^{2+}$ transients $(>1.0 \Delta \mathrm{R} / \mathrm{R})$, but without an egg being successfully released (Fig. 4B,E; Movie 4). These large-amplitude transients that did not lead to egg laying were thus termed "failed egg-laying events." Such failed egg-laying events were infrequent in vulval muscle $\mathrm{Ca}^{2+}$ recordings from WT animals, but they occurred more frequently than successful egg-laying events in transgenic animals expressing TeTx in the VCs (Fig. 4E). Based on these results, it appears that VC neurotransmission does not initiate vulval muscle $\mathrm{Ca}^{2+}$ transients but is instead critical for coordinating vulval muscle $\mathrm{Ca}^{2+}$ activity and contraction across the vulval muscle cells to allow for successful egg release.

Egg laying occurs when strong vulval muscle $\mathrm{Ca}^{2+}$ activity drives the synchronous contraction of all the vulval muscle cells ( $\mathrm{Li}$ et al., 2013; Brewer et al., 2019) that allows for the mechanical opening of the vulva in phase with locomotion for efficient egg release (Collins and Koelle, 2013; Collins et al., 2016). Egg-laying events are characterized by coordinated $\mathrm{Ca}^{2+}$ activity between the vml vulval muscles that extend to the ventral tips of the vulva and the medial vm2 vulval muscles, leading to full contraction and egg release (Fig. 5A). This

muscle opening distance in WT $(\boldsymbol{F})$ and transgenic animals expressing TeTx in the VC neurons $(\boldsymbol{G})$. Lines through points indicate simple linear regression for each labeled grouping. 
A

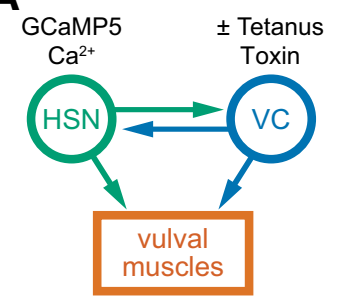

B

\section{$\mathrm{HSN} \mathrm{Ca}{ }^{2+}$}

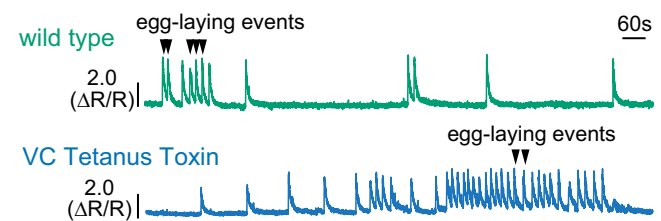

C

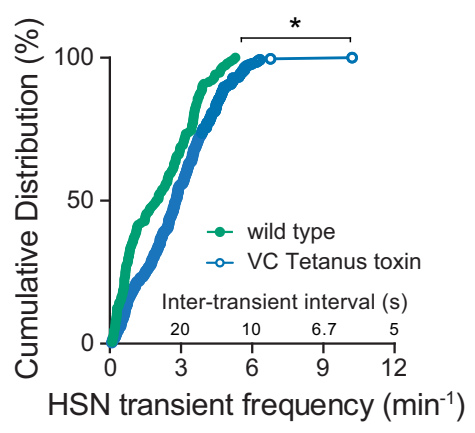

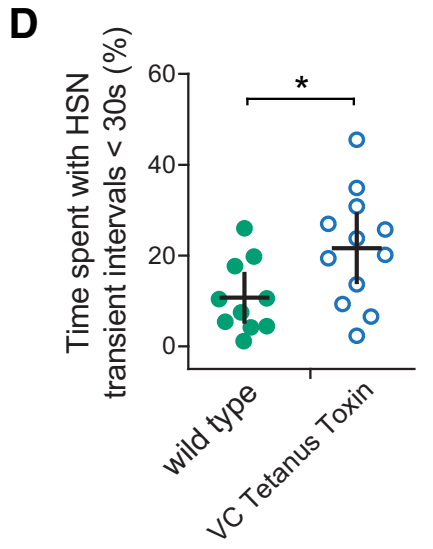

Figure 6. Blocking VC neurotransmission increases $\mathrm{HSN} \mathrm{Ca}^{2+}$ activity. $\boldsymbol{A}$, Diagram of circuit and the experiment. TeTx was expressed in the VC neurons to block their neurotransmitter release, and HSN $\mathrm{Ca}^{2+}$ activity was recorded through GCaMP5 imaging. $\boldsymbol{B}$, Representative traces of HSN neuron GCaMP5/mCherry $\mathrm{Ca}^{2+}$ ratio $(\triangle R / R)$ in a WT background (top, green) and animals expressing TeTx in the VCs (bottom, blue). C, Cumulative distribution plots of the instantaneous frequency of HSN Ca ${ }^{2+}$ transients in WT and TeTx-expressing animals. ${ }^{*} p=0.0006$ (KolmogorovSmirnov test). $n \geq 154$ from $\geq 10$ animals. $\boldsymbol{D}$, Scatterplot for the percentage of time each animal spent with HSN $\mathrm{Ca}^{2+}$ intertransient intervals that were $<30 \mathrm{~s}$, an indicator of hyperactivity. ${ }^{*} p=0.00272$ (Student's $t$ test). $n \geq 10$. Error bars indicate $95 \%$ Cls for the mean.

$\mathrm{Ca}^{2+}$ activity is distinct from weak vulval muscle twitching contractions that are confined to the vm1 muscles (Fig. 5A) (Collins and Koelle, 2013). Failed egg-laying events exhibit $\mathrm{Ca}^{2+}$ activity more similar to egg-laying events, where $\mathrm{Ca}^{2+}$ signal is high across both vm 1 and vm 2 muscles (Fig. 5A). To understand why egg laying was less likely to occur during strong vulval muscle $\mathrm{Ca}^{2+}$ transients in VC neurotransmission-inhibited animals, we measured features of contractile events during egg laying. Contraction can be directly quantified by measuring the reduction in muscle area in fluorescent micrographs, and vulval opening can be quantified by measuring the changing distance between the vulval muscle cells positioned anterior and those posterior to the vulval slit (Fig. 5A). During egg-laying events in WT animals, a strong cytosolic $\mathrm{Ca}^{2+}$ transient correlates with an $\sim 50 \mu \mathrm{m}^{2}$ contraction of muscle size followed by a rebound phase after egg release (Fig. 5B, top and middle). Simultaneously, the anterior and posterior muscles separate by $\sim 10 \mu \mathrm{m}$, facilitating egg release (Fig. 5B, bottom). We found differences in the kinetics and extent of vulval muscle opening between WT and TeTx-expressing transgenic animals during successful egg-laying events (compare Fig. 5B,C). The vulval muscles opened wider, and the degree of contraction was greater during egg-laying events in animals where VC neurotransmission was inhibited with TeTx (Fig. 5C), possibly because the vulval muscle $\mathrm{Ca}^{2+}$ rise started earlier in these animals. During failed egg-laying events, the vulval muscles showed only modest contraction and only separated by $\sim 5 \mu \mathrm{m}$, insufficient to allow egg release, despite reaching similar levels of cytosolic $\mathrm{Ca}^{2+}$ (Fig. 5D). Animals with blocked VC neurotransmission exhibited even less separation of the vulval muscles during failed egg-laying events (compare Fig. 5D and Fig. 5E). In contrast to WT animals, animals with inhibited VC neurotransmission have many more failed egg-laying events during which they exhibit vulval opening kinetics more similar to twitches (compare Fig. $5 F$ and Fig. 5G). To understand the relationship between vulval muscle $\mathrm{Ca}^{2+}$ levels and vulval opening, we measured the distance between anterior and posterior vulval muscles during weak twitching, failed egg-laying events, and successful egg-laying events (Fig. $5 F$ ). In both WT and TeTx-expressing animals, we noted a linear relationship of low but positive slope between vulval opening and $\mathrm{Ca}^{2+}$ levels $<1.0 \Delta \mathrm{R} / \mathrm{R}\left(\Delta\right.$ opening/ $\Delta \mathrm{Ca}^{2+}$ ) during weak twitching contractions (Fig. 5F,G). However, as $\mathrm{Ca}^{2+}$ levels rose above $1.0 \Delta \mathrm{R} / \mathrm{R}$, the muscles reached threshold for full opening, allowing successful egg release (Fig. 5F). The steep, linear $\Delta$ opening/ $\Delta \mathrm{Ca}^{2+}$ relationship during failed egg-laying events suggests that a threshold of $\mathrm{Ca}^{2+}$ drives an all-or-none transition to full contraction, vulval opening, and egg release. In VC neurotransmission-inhibited animals, the shallow $\Delta$ opening $/ \Delta \mathrm{Ca}^{2+}$ relationship continued as weak twitches transitioned into failed egg-laying events, with many strong vulval muscle $\mathrm{Ca}^{2+}$ transients failing to open the vulva sufficiently for egg release (Fig. 5G). However, successful egg-laying events in VC neurotransmission-inhibited animals still showed a sharp threshold between $\mathrm{Ca}^{2+}$ levels and the degree of vulval opening. This raises the possibility of two types of failed egg-laying events: one that is shared with WT animals, and another that is unique to animals with inhibited VC neurotransmission.

\section{VC neurotransmission regulates HSN command neuron and egg-laying circuit activity}

To determine whether VC synaptic transmission regulates egg laying via $\mathrm{HSN}$, we recorded $\mathrm{HSN} \mathrm{Ca}^{2+}$ activity in WT and transgenic animals expressing TeTx in the VCs (Fig. 6A). During the egg-laying active state, the HSNs drive egg laying during periods of increased $\mathrm{Ca}^{2+}$ transient frequency in the form of burst firing (Fig. 6B) (Collins et al., 2016; Ravi et al., 2018a). We observed a significant increase in $\mathrm{HSN} \mathrm{Ca}{ }^{2+}$ transient frequency when VC synaptic transmission was blocked compared with nontransgenic control animals (Fig. 6C). WT animals spent $\sim 11 \%$ of their time exhibiting high-frequency burst activity in the HSN neurons, whereas transgenic animals expressing TeTx in the VC neurons spent $\sim 21 \%$ of their time exhibiting HSN burst firing activity (Fig. 6D). These results are consistent with the interpretation that VC neurotransmission is inhibitory toward the HSNs, such as proposed in previous studies (Bany et al., 2003; Zhang et al., 2008), but the steady-state egg accumulation of animals expressing VC-specific TeTx or $\mathrm{HisCl}$ is normal (Fig. $1 G, K)$. We have previously shown that HSN burst firing is regulated by egg accumulation and feedback of vulval muscle $\mathrm{Ca}^{2+}$ activity (Ravi et al., 2018a), which could be enhanced by the high rate of failed egg-laying events observed in VC TeTx transgenic animals (Fig. 5E). We propose that the increased HSN burst firing seen in VC TeTx transgenic animals does not solely result from loss of inhibitory $\mathrm{VC}$ input, but also reflects the 
A

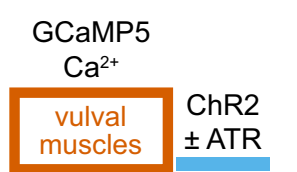

C Vulval muscle ChR2, $\mathrm{Ca}^{2+}$
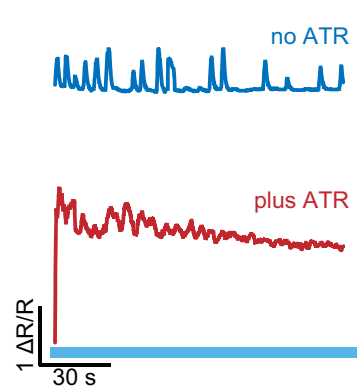

D

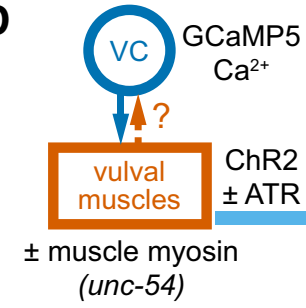

F Vulval muscle ChR2, $\mathrm{VC} \mathrm{Ca}^{2+}$

no ATR
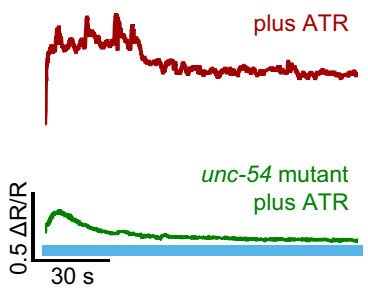

G

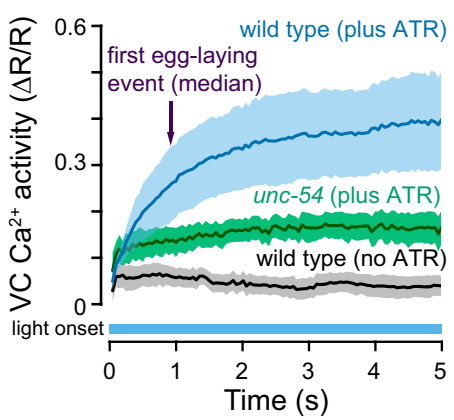

E
B Vulval muscle ChR2, vulval muscle $\mathrm{Ca}^{2+}$
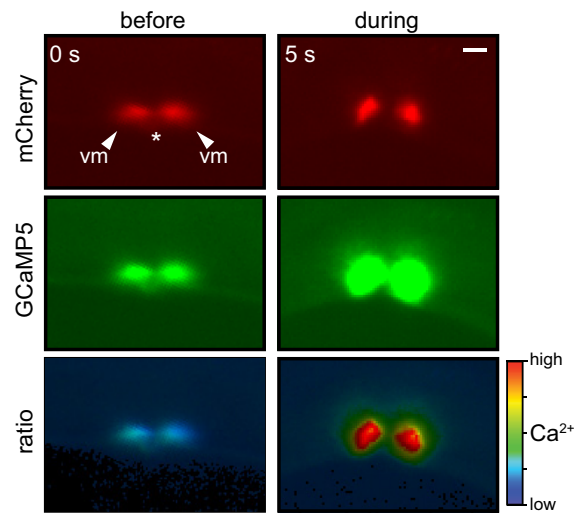

E Vulval muscle ChR2, VC neuron $\mathrm{Ca}^{2+}$
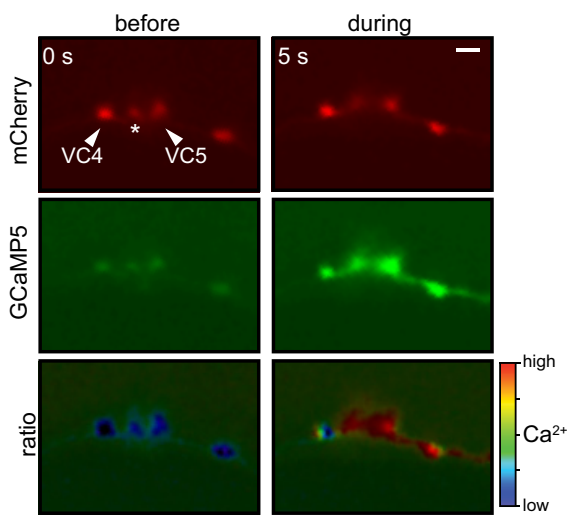

H

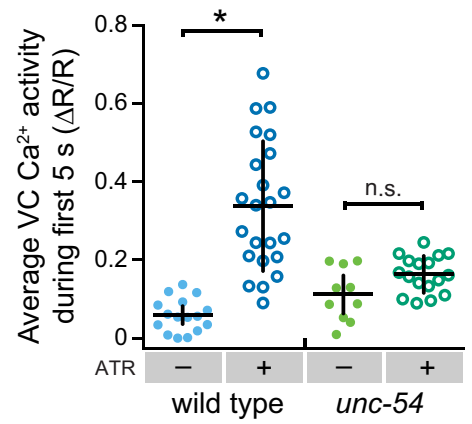

Figure 7. Optogenetic activation and contraction of the vulval muscles drive VC neuron activity. $A$, Diagram of experiment. ChR2 and GCaMP5 were expressed in the vulval muscles to monitor cytosolic $\mathrm{Ca}^{2+}$ after optogenetic stimulation. $\boldsymbol{B}$, Representative still images of vulval muscle mCherry, GCaMP5, and GCaMP5/mCherry ratio during optogenetic activation of the vulval muscles. Arrowheads indicate each vulval muscle half. *Vulva. Scale bar, $20 \mu \mathrm{m}$. C, Representative traces of vulval muscle GCaMP5/mCherry $\mathrm{Ca}^{2+}$ ratio $(\triangle \mathrm{R} / \mathrm{R})$ in animals expressing $\mathrm{ChR2}$ in the vulval muscles in the absence (-ATR, top) or presence (+ATR, bottom) of ATR during 3 min of continuous blue light exposure. D, Diagram of circuit and experiment. ChR2 was expressed in the vulval muscles, and GCaMP5 was expressed in the VC neurons to record $\mathrm{Ca}^{2+}$ activity in WT or unc-54 myosin null mutant animals. $\boldsymbol{E}$, Representative still images of VC neuron mCherry, GCaMP5, and GCaMP5/mCherry ratio during optogenetic activation of the vulval muscles. Arrowheads indicate VC neuron cell bodies. *Vulva. Scale bar, $20 \mu \mathrm{m}$. $\boldsymbol{F}$, Representative traces of VC neuron GCaMP5/

circuit prolonging the active state to compensate for more failed egg-laying transients in the absence of excitatory VC input.

The VC motor neurons are responsive to vulval muscle activity and contraction $\mathrm{VC} \mathrm{Ca}{ }^{2+}$ activity is coincident with strong vulval muscle twitching and egg-laying contractions (Fig. 2) (Collins et al., 2016). In addition to making synapses onto the vm2 muscles whose contraction drives egg laying, the VCs extend neurites along the vulval hypodermis devoid of synapses (White et al., 1986), suggesting that the VCs may respond to vulval opening. To test this model, we sought to induce vulval opening independent of endogenous circuit activity and presynaptic input from the HSNs. We transgenically expressed ChR2 specifically in the vulval muscles using the ceh-24 promoter to stimulate the vulval muscles and used GCaMP5 to record blue-light induced changes in vulval muscle $\mathrm{Ca}^{2+}$ activity (Fig. $7 A$ ). Optogenetic stimulation of the vulval muscles triggered an immediate rise in vulval muscle cytosolic $\mathrm{Ca}^{2+}$, tonic contraction of the vulval muscles, vulval opening, and egg release (Fig. $7 B, C$ ). Although optogenetic stimulation resulted in sustained vulval muscle $\mathrm{Ca}^{2+}$ activity and contraction, vulval opening and egg release remained rhythmic and phased with locomotion, as previously observed in WT animals (Collins and Koelle, 2013; Collins et al., 2016). Simultaneous brightfield recordings showed the vulva only opened for egg release when the adjacent ventral body wall muscles were in a relaxed phase (Movie 5). We have previously shown that eggs are preferentially released when the vulva is at a particular phase of the body bend, typically as the ventral body wall muscles anterior to the vulva go into a more relaxed state (Collins and Koelle, 2013; Collins et al., 2016). We now interpret this phasing of egg release with locomotion as evidence that vulval muscle $\mathrm{Ca}^{2+}$ activity drives contraction, but the vulva only opens for successful egg release when contraction is initiated during relaxation of the adjacent body wall muscles. Together, these results show that optogenetic stimulation of the vulval muscles is sufficient to induce vulval muscle $\mathrm{Ca}^{2+}$ activity for egg release in a locomotion phase-dependent manner.

mCherry $\mathrm{Ca}^{2+}$ ratio $(\Delta \mathrm{R} / \mathrm{R})$ in animals expressing $(\mathrm{CR} 2$ in the vulval muscles in the absence (-ATR, top) or presence (+ATR, bottom) of ATR during $3 \mathrm{~min}$ of continuous blue light exposure. $\mathbf{G}$, Averaged VC $\mathrm{Ca}^{2+}$ responses during the first $5 \mathrm{~s}$. Error bands represent \pm 95 Cls for the mean; $n \geq 10$ animals. $\boldsymbol{H}$, Scatterplot represents the average $\mathrm{VC} \mathrm{Ca}^{2+}$ response ( $\pm 95 \mathrm{Cls}$ ) during the first $5 \mathrm{~s}$ of optogenetic stimulation of the vulval muscles. $* p<0.0001$. n.s. indicates $p>0.05$ (one-way ANOVA with Bonferroni's correction for multiple comparisons). $n \geq 10$ animals. 


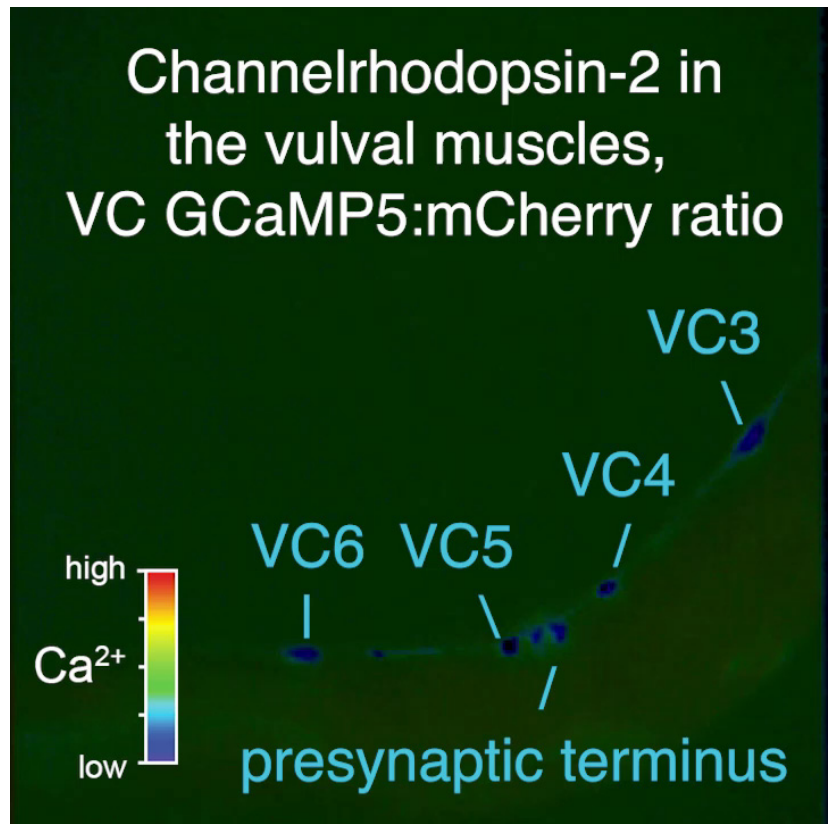

Movie 5. GCaMP5/mCherry ratio recording of $\mathrm{VC}^{2} \mathrm{C}^{2+}$ in response to optogenetic stimulation of the vulval muscles. Ratio of GCaMP5 and mCherry fluorescence in the VCs mapped onto a false color spectrum ranging from blue (low) to red (high). Optogenetic activation of the vulval muscles causes an immediate induction of $\mathrm{VCCa}^{2+}$ activity that remains at a high level for the duration of the stimulation. [View online]

To test the hypothesis that the VCs respond to vulval muscle activation, we recorded changes in $\mathrm{VC} \mathrm{Ca}^{2+}$ on optogenetic stimulation of the vulval muscles (Fig. $7 D$ ). We observed a robust induction of $\mathrm{VC} \mathrm{Ca}^{2+}$ activity on blue light illumination (Fig. $7 E, F)$. The rise in $\mathrm{VC} \mathrm{Ca}^{2+}$ occurred before the first egg-laying event, suggesting that this process is dependent on muscle activity and not necessarily the passage of an egg (Fig. 7G; Movie 5). This result demonstrates that the VCs can become excited in response to activity of the postsynaptic vulval muscles.

How do the vulval muscles activate the VCs? The VCs make both chemical and electrical synapses onto the vm2 vulval muscles (White et al., 1986; Cook et al., 2019). Depolarization of the vm2 vulval muscles might be expected to electrically propagate to the $\mathrm{VC}$ and trigger an increase in $\mathrm{VC} \mathrm{Ca}{ }^{2+}$ activity. Another possibility is that the VCs are mechanically activated in response to vulval muscle contraction and/or vulval opening. To test these alternate models, we optogenetically stimulated the vulval muscles of unc- 54 muscle myosin mutants, which are unable to contract their muscles, and recorded $\mathrm{VC} \mathrm{Ca}^{2+}$ activity (Fig. 7D). We found optogenetic activation of the vulval muscles failed to induce $\mathrm{VC} \mathrm{Ca}^{2+}$ activity in unc-54 mutants compared with the WT background (Fig. 7G,H). While unc-54 mutants appear to show some increase in $\mathrm{VC} \mathrm{Ca}^{2+}$ activity following blue light stimulation of the vulval muscles, this increase was not statistically significant, suggesting indirect excitation of the VCs through gap junctions is insufficient on its own to induce robust $\mathrm{VC} \mathrm{Ca}{ }^{2+}$ activity. Together, these results support a model where the VC neurons are mechanically activated in response to vulval muscle contraction. Mechanical activation appears to drive VC activity and is mediated through the VC4 and VC5 neurites that are most proximal to the vulval canal through which eggs are laid.

\section{Discussion}

The connectome of C. elegans has greatly informed neural circuit studies and contributed to studies revealing that connectivity

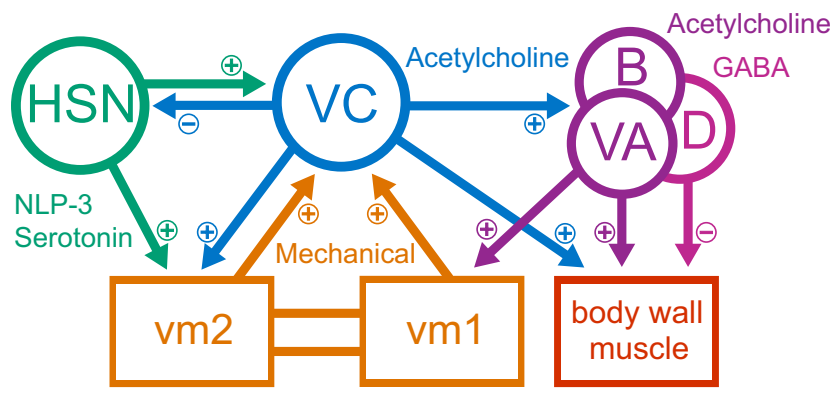

Figure 8. The VC neurons function to coordinate synchronized contraction of the vulval muscles for egg laying in response to serotonin-mediated potentiation. Working model of the functional connectivity within the C. elegans egg-laying circuit (White et al., 1986; Cook et al., 2019). +, Excitatory input; -, inhibitory input. HSN command neurons release serotonin and NLP-3 to potentiate the VCs and vm2 vulval muscles (see Figs. 1, 2) (Waggoner et al., 1998; Shyn et al., 2003; Zhang et al., 2008; Collins et al., 2016). VC neurons release ACh that excites the vm2 vulval muscles, body wall muscles, and the VA/VB/VD locomotion motor neurons (Fig. 3) (Kim et al., 2001; Collins et al., 2016), as well as ACh to inhibit the HSNs (Fig. 6) (Bany et al., 2003; Zhang et al., 2008). The VA/NB/ND neurons release ACh and GABA onto the body wall muscles to regulate locomotion (Richmond and Jorgensen, 1999; Zhen and Samuel, 2015), and onto the vm1 vulval muscles to initiate contractions for egg laying (Collins and Koelle, 2013; Collins et al., 2016). Contraction of the vm1 vulval muscles electrically excites the vm2 vulval muscles and mechanically activates the VCs (Fig. 7), forming a positive feedback loop until all vulval muscle cells are contracted and egg laying occurs.

alone is not sufficient to explain nervous system operations (Bargmann, 2012; Bentley et al., 2016). In the present study, we examined the neural circuit driving egg-laying behavior in C. elegans at a cellular resolution to reveal functional pathways and elements of the behavior that had not been discernable through connectome or prior genetic studies (Fig. 8). We show that the cholinergic VC motor neurons contribute to egg laying in a serotonergic pathway. Our data show that HSN activity acts to excite the vulval muscles and VCs, and that $\mathrm{VC} \mathrm{Ca}^{2+}$ activity is closely associated with visible vulval muscle contraction. In the absence of HSN-mediated potentiation, the VCs are able to excite the vulval muscles, but not to the threshold required for egg laying. This subthreshold interaction is consistent with a model where serotonin-mediated potentiation of the VCs and vulval muscles is required before the VCs can facilitate egg laying through timely excitatory input. In the absence of VC neurotransmission, the HSNs and the vulval muscles show excess $\mathrm{Ca}^{2+}$ activity, but this excess activity does not correspond to increased egg laying. Instead, we find that the vulval muscles are less efficient at opening the vulva, indicating that the VCs have a role in facilitating vulval muscle contraction. Surprisingly, optogenetic activation and contraction of the postsynaptic vulval muscles are sufficient to drive presynaptic $\mathrm{VC} \mathrm{Ca}^{2+}$ activity. We propose that serotonin released from the HSNs signals to promote both vulval muscle contractility and VC sensitivity to that contraction. Following this potentiation, vulval muscle twitch contractions are able to mechanically activate the VCs to release excitatory $\mathrm{ACh}$ as part of a positive-feedback loop until the vulval muscles are fully contracted and egg laying occurs.

Many behaviors require downstream feedback to help finetune movements and make adjustments based on the changing internal and external environment, such as with wing-beat patterns in Drosophila or head-eye coordination in humans (Fang et al., 2015; Bartussek and Lehmann, 2016). We find that the downstream target of the egg-laying neural circuit, the vulval muscles, signals upstream to facilitate proper completion of the behavior. We postulate that such feedback signaling is critical for two reasons. First, it can act to feedback inhibit the circuit to signal 
when the behavior has been executed. We find that vulval muscle contraction activates the VCs (Fig. 7), which could contribute to inhibition of the egg-laying circuit through release of ACh acting on metabotropic receptors, such as GAR-2 on the HSNs and vulval muscles (Bany et al., 2003; Zhang et al., 2008, 2010; Fernandez et al., 2020). GAR-2 has previously been shown to act in parallel with ionotropic receptors to differentially modulate locomotion behavior, providing both short- and longer-term effects in response to cholinergic signaling (Dittman and Kaplan, 2008; Zhen and Samuel, 2015). Second, feedback signaling could create a positive feedback loop to facilitate full execution of a behavior. In this circuit model, the VCs are mechanically activated by vulval muscle twitches, which in turn leads to the VCs further exciting the vulval muscles until ubiquitous and coordinated contraction is achieved. A similar type of feedback activity has been demonstrated in the C. elegans SMDD neurons where TRPC channels, TRP-1 and TRP-2, are mechanically activated by neck muscle contractions to influence neck steering behavior (Yeon et al., 2018), as well as the crab cardiac ganglion where mechanosensitive dendrites receive feedback from the postsynaptic muscles to modify the ganglion's firing activity (GarcíaCrescioni et al., 2010). Which receptors mediate VC mechanosensation is not known, but the VCs do express innexin gap junction proteins (Altun et al., 2009) that have recently been shown to act as mechanosensitive hemichannels (Walker and Schafer, 2020).

Serotonergic modulation of neural circuits and behavior is a well-characterized phenomenon across both invertebrate and vertebrate animals (Weiger, 1997; Bacqué-Cazenave et al., 2020). In the mouse nociceptive circuit, serotonergic modulation has been shown to confer hyperalgesia (Lin et al., 2011; Bardoni, 2019). In this circuit, serotonin acts on DRG neurons to increase their sensitivity to mechanical stimuli through the $5-\mathrm{HT}_{2 \mathrm{~B}}$ receptor ( $\mathrm{Su}$ et al., 2016) and to maintain this sensitivity through $5-\mathrm{HT}_{4 / 6 / 7}$ receptors (Godínez-Chaparro et al,, 2011). In the C. elegans egg-laying circuit, serotonin released by the HSN command neurons signals through several G-protein coupled receptors, including the $5-\mathrm{HT}_{2}$ ortholog SER-1 and the $5-\mathrm{HT}_{7}$ ortholog SER-7 expressed on the vulval muscles (Hobson et al., 2006; Xiao et al., 2006; Hapiak et al., 2009). These serotonin receptors are thought to act through $\mathrm{Ga}_{\mathrm{q}}$ and $\mathrm{Ga}_{\mathrm{s}}$ signaling pathways, respectively, which activate EGL-19 L-type voltage-gated $\mathrm{Ca}^{2+}$ channels in the vulval muscles to enhance their response to other excitatory input (Waggoner et al., 1998; Schafer, 2006; Zhang et al., 2008). SER-7 is also expressed in the VC neurons (Fernandez et al., 2020), and serotonin acting through SER-7 has been shown to initiate motor neurons activity in other behaviors, such as feeding (Song et al., 2013). Like animals with blocked VC neurotransmission (Fig. 1), ser-7 mutants also fail to respond to exogenous serotonin (Hobson et al., 2006). Downstream targets of serotonergic signaling onto the VCs may include the N/P/Qtype $\mathrm{Ca}^{2+}$ channel UNC-2 to promote neurotransmitter release (Schafer et al., 1996). Following serotonergic potentiation, the VCs may be close enough to threshold to become mechanically excited in response to vm1-mediated vulval muscle twitches, leading to excitatory $\mathrm{VC}$ neurotransmitter release onto the vm2 vulval muscles to drive complete vulval muscle contraction for egg release.

Synaptic wiring diagrams show multiple sites of cross-innervation between the canonical egg-laying circuit and locomotion circuit (White et al., 1986; Cook et al., 2019). Consistent with this shared connectivity, egg-laying behavior is correlated with changes in locomotion behavior (Hardaker et al., 2001; Collins et al., 2016), suggesting that these two circuits actively communicate and coordinate their respective behaviors. Coordination of distinct neural circuits and behaviors has been demonstrated in the stomatogastric ganglion of the lobster, where two overlapping circuits are phase-coordinated with one another to regulate gastric peristalsis and subsequent digestion (Clemens et al., 1998; Bartos et al., 1999). The VCs have been shown to regulate locomotion, potentially through the GABAergic motor neurons or via direct release of $\mathrm{ACh}$ that drives excitation and contraction of the body wall muscles to slow locomotion (Fig. 8) (Collins et al., 2016). Slowing of locomotion during egg laying may provide time for the vulval muscles to fully contract (Collins and Koelle, 2013; Collins et al., 2016). This slowing could function to hold the animal in a body posture that is favorable for vulval opening and egg release, which would be consistent with our finding that VC activity causes elongated vulval muscle $\mathrm{Ca}^{2+}$ twitch transients (Fig. 3). In this role, the VCs would be signaling to the rest of the egg-laying circuit and locomotion circuit that the vulva is open so that body posture can be held in a favorable phase for egg release. The six VCs also make synapses onto each another (Cook et al., 2019). The mechanical activation of the vulva-proximal VCs during vulval opening may excite the distal VC neurons to slow locomotion and control body posture for efficient egg release. Failed egg-laying events could occur because of disrupted phasing of the locomotion pattern with activity in the egg-laying circuit. Since twitches and egg-laying events occur at a specific phase of the locomotion pattern (Collins and Koelle, 2013), the opening of the vulva and egg release may require not only coordinated muscle contraction, but also the relaxation of immediately proximal body wall muscles so that they cannot physically resist the full opening of the vulva. Thus, the VCs may act to coordinate the excitation of the vm2 muscles with excitatory input from the VA/VB locomotion motor neurons onto the vm1 muscles to facilitate full contraction and egg laying in phase with locomotion.

In conclusion, the VC neurons and the egg-laying circuit present a tractable model system for investigating how different forms of chemical signaling and mechanosensory feedback work together to drive a robust behavior. The elucidation of the cellular and molecular mechanisms underlying these distinct forms of feedback could help the understanding of human neurologic diseases where muscle coordination and proprioception are dysregulated, such as in Parkinson's and Huntington disease (Bargmann, 2012; Lukos et al., 2013).

\section{References}

Altun ZF, Chen B, Wang ZW, Hall DH (2009) High resolution map of Caenorhabditis elegans gap junction proteins. Dev Dyn 238:1936-1950.

Bacqué-Cazenave J, Bharatiya R, Barrière G, Delbecque JP, Bouguiyoud N, Di Giovanni G, Cattaert D, De Deurwaerdère P (2020) Serotonin in animal cognition and behavior. Int J Mol Sci 21:1649.

Banerjee N, Bhattacharya R, Gorczyca M, Collins KM, Francis MM (2017) Local neuropeptide signaling modulates serotonergic transmission to shape the temporal organization of C. elegans egg-laying behavior. PLoS Genet 13:e1006697.

Bany IA, Dong MQ, Koelle MR (2003) Genetic and cellular basis for acetylcholine inhibition of Caenorhabditis elegans egg-laying behavior. J Neurosci 23:8060-8069.

Bardoni R (2019) Serotonergic modulation of nociceptive circuits in spinal cord dorsal horn. Curr Neuropharmacol 17:1133-1145.

Bargmann CI (2012) Beyond the connectome: how neuromodulators shape neural circuits. Bioessays 34:458-465.

Bargmann CI, Marder E (2013) From the connectome to brain function. Nat Methods 10:483-490. 
Bartos M, Manor Y, Nadim F, Marder E, Nusbaum MP (1999) Coordination of fast and slow rhythmic neuronal circuits. J Neurosci 19:6650-6660.

Bartussek J, Lehmann FO (2016) Proprioceptive feedback determines visuomotor gain in Drosophila. R Soc Open Sci 3:150562.

Batista-García-Ramó K, Fernández-Verdecia CI (2018) What we know about the brain structure-function relationship. Behav Sci (Basel) 8:39.

Bentley B, Branicky R, Barnes CL, Chew YL, Yemini E, Bullmore ET, Vértes PE, Schafer WR (2016) The multilayer connectome of Caenorhabditis elegans. PLoS Comput Biol 12:e1005283.

Brenner S (1974) The genetics of Caenorhabditis elegans. Genetics 77:71-94.

Brewer JC, Olson AC, Collins KM, Koelle MR (2019) Serotonin and neuropeptides are both released by the HSN command neuron to initiate Caenorhabditis elegans egg laying. PLoS Genet 15:e1007896.

Chase DL, Pepper JS, Koelle MR (2004) Mechanism of extrasynaptic dopamine signaling in Caenorhabditis elegans. Nat Neurosci 7:1096-1103.

Clark SG, Lu X, Horvitz HR (1994) The Caenorhabditis elegans locus lin-15, a negative regulator of a tyrosine kinase signaling pathway, encodes two different proteins. Genetics 137:987-997.

Clemens S, Combes D, Meyrand P, Simmers J (1998) Long-term expression of two interacting motor pattern-generating networks in the stomatogastric system of freely behaving lobster. J Neurophysiol 79:1396-1408.

Collins KM, Koelle MR (2013) Postsynaptic ERG potassium channels limit muscle excitability to allow distinct egg-laying behavior states in Caenorhabditis elegans. J Neurosci 33:761-775.

Collins KM, Bode A, Fernandez RW, Tanis JE, Brewer JC, Creamer MS, Koelle MR (2016) Activity of the C. elegans egg-laying behavior circuit is controlled by competing activation and feedback inhibition. Elife 5: e21126.

Cook SJ, Jarrell TA, Brittin CA, Wang Y, Bloniarz AE, Yakovlev MA, Nguyen KC, Tang LT, Bayer EA, Duerr JS, Bülow HE, Hobert O, Hall DH, Emmons SW (2019) Whole-animal connectomes of both Caenorhabditis elegans sexes. Nature 571:63-71.

Del-Bel E, De-Miguel FF (2018) Extrasynaptic neurotransmission mediated by exocytosis and diffusive release of transmitter substances. Front Synaptic Neurosci 10:13.

Desai C, Garriga G, McIntire SL, Horvitz HR (1988) A genetic pathway for the development of the Caenorhabditis elegans HSN motor neurons. Nature 336:638-646.

Dittman JS, Kaplan JM (2008) Behavioral impact of neurotransmitter-activated G-protein-coupled receptors: muscarinic and GABAB receptors regulate Caenorhabditis elegans locomotion. J Neurosci 28:7104-7112.

Donnelly JL, Clark CM, Leifer AM, Pirri JK, Haburcak M, Francis MM, Samuel ADT, Alkema MJ (2013) Monoaminergic orchestration of motor programs in a complex C. elegans behavior. PLoS Biol 11:e1001529.

Duerr JS, Gaskin J, Rand JB (2001) Identified neurons in C. elegans coexpress vesicular transporters for acetylcholine and monoamines. Am J Physiol Cell Physiol 280:C1616-C1622.

Duerr JS, Han HP, Fields SD, Rand JB (2008) Identification of major classes of cholinergic neurons in the nematode Caenorhabditis elegans. J Comp Neurol 506:398-408.

Emtage L, Aziz-Zaman S, Padovan-Merhar O, Horvitz HR, Fang-Yen C, Ringstad N (2012) IRK-1 potassium channels mediate peptidergic inhibition of Caenorhabditis elegans serotonin neurons via a go signaling pathway. J Neurosci 32:16285-16295.

Fang Y, Nakashima R, Matsumiya K, Kuriki I, Shioiri S (2015) Eye-head coordination for visual cognitive processing. PLoS One 10:e0121035.

Fernandez RW, Wei K, Wang EY, Mikalauskaite D, Olson A, Pepper J, Christie N, Kim S, Koelle MR (2020) Cellular expression and functional roles of all 26 neurotransmitter GPCRs in the C. elegans egg-laying circuit. BioRxiv 04.23.037242.

García-Crescioni K, Fort TJ, Stern E, Brezina V, Miller MW (2010) Feedback from peripheral musculature to central pattern generator in the neurogenic heart of the crab Callinectes sapidus: role of mechanosensitive dendrites. J Neurophysiol 103:83-96.

Godínez-Chaparro B, Barragán-Iglesias P, Castañeda-Corral G, RochaGonzález HI, Granados-Soto V (2011) Role of peripheral 5-HT(4), 5-HT (6), and 5-HT(7) receptors in development and maintenance of secondary mechanical allodynia and hyperalgesia. Pain 152:687-697.

Hapiak VM, Hobson RJ, Hughes L, Smith K, Harris G, Condon C, Komuniecki P, Komuniecki RW (2009) Dual excitatory and inhibitory serotonergic inputs modulate egg laying in Caenorhabditis elegans. Genetics 181:153-163.
Hardaker LA, Singer E, Kerr R, Zhou G, Schafer WR (2001) Serotonin modulates locomotory behavior and coordinates egg-laying and movement in Caenorhabditis elegans. J Neurobiol 49:303-313.

Hardingham GE, Bading H (2010) Synaptic versus extrasynaptic NMDA receptor signalling: implications for neurodegenerative disorders. Nat Rev Neurosci 11:682-696.

Hobson RJ, Hapiak VM, Xiao H, Buehrer KL, Komuniecki PR, Komuniecki RW (2006) SER-7, a Caenorhabditis elegans 5-HT7-like receptor, is essential for the 5-HT stimulation of pharyngeal pumping and egg laying. Genetics 172:159-169.

Jose AM, Bany IA, Chase DL, Koelle MR (2007) A specific subset of transient receptor potential vanilloid-type channel subunits in Caenorhabditis elegans endocrine cells function as mixed heteromers to promote neurotransmitter release. Genetics 175:93-105.

Kim J, Poole DS, Waggoner LE, Kempf A, Ramirez DS, Treschow PA, Schafer WR (2001) Genes affecting the activity of nicotinic receptors involved in Caenorhabditis elegans egg-laying behavior. Genetics 157:1599-1610.

Koelle MR (2018) Neurotransmitter signaling through heterotrimeric G proteins: insights from studies in C. elegans. WormBook 2018:1-52.

Koelle MR, Horvitz HR (1996) EGL-10 regulates G protein signaling in the C. elegans nervous system and shares a conserved domain with many mammalian proteins. Cell 84:115-125.

Lerner TN, Ye L, Deisseroth K (2016) Communication in neural circuits: tools, opportunities, and challenges. Cell 164:1136-1150.

Li P, Collins KM, Koelle MR, Shen K (2013) LIN-12/Notch signaling instructs postsynaptic muscle arm development by regulating UNC-40/ DCC and MADD-2 in Caenorhabditis elegans. Elife 2:e00378.

Lin SY, Chang WJ, Lin CS, Huang CY, Wang HF, Sun WH (2011) Serotonin receptor 5-HT2B mediates serotonin-induced mechanical hyperalgesia. J Neurosci 31:1410-1418.

Liu P, Chen B, Wang ZW (2013) Postsynaptic current bursts instruct action potential firing at a graded synapse. Nat Commun 4:1911.

Liu Q, Hollopeter G, Jorgensen EM (2009) Graded synaptic transmission at the Caenorhabditis elegans neuromuscular junction. Proc Natl Acad Sci USA 106:10823-10828.

Lukos JR, Snider J, Hernandez ME, Tunik E, Hillyard S, Poizner H (2013) Parkinson's disease patients show impaired corrective grasp control and eye-hand coupling when reaching to grasp virtual objects. Neuroscience 254:205-221.

Meinertzhagen IA (2018) Of what use is connectomics? A personal perspective on the Drosophila connectome. J Exp Biol 221:jeb164954.

Nusbaum MP, Blitz DM, Marder E (2017) Functional consequences of neuropeptide and small-molecule co-transmission. Nat Rev Neurosci 18:389-403.

Pereira L, Kratsios P, Serrano-Saiz E, Sheftel H, Mayo AE, Hall DH, White JG, LeBoeuf B, Garcia LR, Alon U, Hobert O (2015) A cellular and regulatory map of the cholinergic nervous system of C. elegans. Elife 4:e12432.

Pokala N, Liu Q, Gordus A, Bargmann CI (2014) Inducible and titratable silencing of Caenorhabditis elegans neurons in vivo with histamine-gated chloride channels. Proc Natl Acad Sci USA 111:2770-2775.

Ravi B, Garcia J, Collins KM (2018a) Homeostatic feedback modulates the development of two-state patterned activity in a model serotonin motor circuit in Caenorhabditis elegans. J Neurosci 38:6283-6298.

Ravi B, Nassar LM, Kopchock RJ III, Dhakal P, Scheetz M, Collins KM (2018b) Ratiometric calcium imaging of individual neurons in behaving Caenorhabditis elegans. J Vis Exp 132:e56911.

Richmond JE, Jorgensen EM (1999) One GABA and two acetylcholine receptors function at the C. elegans neuromuscular junction. Nat Neurosci 2:791-797.

Schafer WR (2006) Genetics of egg-laying in worms. Annu Rev Genet 40:487-509.

Schafer WR, Sanchez BM, Kenyon C (1996) Genes affecting sensitivity to serotonin in Caenorhabditis elegans. Genetics 143:1219-1230.

Schindelin J, Arganda-Carreras I, Frise E, Kaynig V, Longair M, Pietzsch T, Preibisch S, Rueden C, Saalfeld S, Schmid B, Tinevez JY, White DJ, Hartenstein V, Eliceiri K, Tomancak P, Cardona A (2012) Fiji: an opensource platform for biological-image analysis. Nat Methods 9:676-682.

Sengupta P, Samuel AD (2009) C. elegans: a model system for systems neuroscience. Curr Opin Neurobiol 19:637-643. 
Shyn SI, Kerr R, Schafer WR (2003) Serotonin and go modulate functional states of neurons and muscles controlling C. elegans egg-laying behavior. Curr Biol 13:1910-1915.

Song B, Faumont S, Lockery S, Avery L (2013) Recognition of familiar food activates feeding via an endocrine serotonin signal in Caenorhabditis elegans. Elife 2:e00329.

Su YS, Chiu YY, Lin SY, Chen CC, Sun WH (2016) Serotonin receptor 2B mediates mechanical hyperalgesia by regulating transient receptor potential vanilloid 1. J Mol Neurosci 59:113-125.

Swanson LW, Lichtman JW (2016) From Cajal to connectome and beyond. Annu Rev Neurosci 39:197-216.

Taylor SR, Santpere G, Reilly M, Glenwinkel L, Poff A, McWhirter R, Xu C, Weinreb A, Basavaraju M, Cook SJ, Barrett A, Abrams A, Vidal B, Cros C, Rafi I, Sestan N, Hammarlund M, Hobert O, Miller DM (2019) Expression profiling of the mature C. elegans nervous system by singlecell RNA-Sequencing. BioRxiv 737577.

Trent C, Tsuing N, Horvitz HR (1983) Egg-laying defective mutants of the nematode Caenorhabditis elegans. Genetics 104:619-647.

Trojanowski NF, Raizen DM, Fang-Yen C (2016) Pharyngeal pumping in Caenorhabditis elegans depends on tonic and phasic signaling from the nervous system. Sci Rep 6:22940.

Waggoner LE, Zhou GT, Schafer RW, Schafer WR (1998) Control of alternative behavioral states by serotonin in Caenorhabditis elegans. Neuron 21:203-214.

Waggoner LE, Dickinson KA, Poole DS, Tabuse Y, Miwa J, Schafer WR (2000) Long-term nicotine adaptation in Caenorhabditis elegans involves PKC-dependent changes in nicotinic receptor abundance. J Neurosci 20:8802-8811.
Walker DS, Schafer WR (2020) Distinct roles for innexin gap junctions and hemichannels in mechanosensation. Elife 9:e50597.

Weiger WA (1997) Serotonergic modulation of behaviour: a phylogenetic overview. Biol Rev Camb Philos Soc 72:61-95.

Whim MD, Niemann H, Kaczmarek LK (1997) The secretion of classical and peptide cotransmitters from a single presynaptic neuron involves a synaptobrevin-like molecule. J Neurosci 17:2338-2347.

White JG, Southgate E, Thomson JN, Brenner S (1986) The structure of the nervous system of the nematode Caenorhabditis elegans. Philos Trans $\mathrm{R}$ Soc Lond B Biol Sci 314:1-340.

Xiao H, Hapiak VM, Smith KA, Lin L, Hobson RJ, Plenefisch J, Komuniecki R (2006) SER-1, a Caenorhabditis elegans 5-HT2-like receptor, and a multi-PDZ domain containing protein (MPZ-1) interact in vulval muscle to facilitate serotonin-stimulated egg-laying. Dev Biol 298:379-391.

Yeon J, Kim J, Kim DY, Kim H, Kim J, Du EJ, Kang K, Lim HH, Moon D, Kim K (2018) A sensory-motor neuron type mediates proprioceptive coordination of steering in C. elegans via two TRPC channels. PLoS Biol 16:e2004929.

Zhang M, Chung SH, Fang-Yen C, Craig C, Kerr RA, Suzuki H, Samuel AD, Mazur E, Schafer WR (2008) A self-regulating feed-forward circuit controlling C. elegans egg-laying behavior. Curr Biol 18:1445-1455.

Zhang M, Schafer WR, Breitling R (2010) A circuit model of the temporal pattern generator of Caenorhabditis egg-laying behavior. BMC Syst Biol $4: 81$

Zhen M, Samuel AD (2015) C. elegans locomotion: small circuits, complex functions. Curr Opin Neurobiol 33:117-126. 